\title{
VLTI-PIONIER imaging of the red supergiant V602 Carinae ${ }^{\star}$
}

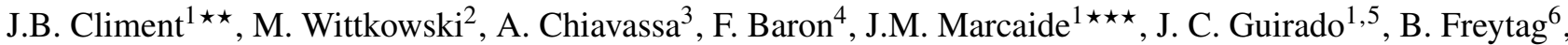 \\ S. Höfner ${ }^{6}$, X. Haubois ${ }^{7}$, and J. Woillez ${ }^{2}$ \\ 1 Departament d'Astronomia i Astrofísica, Universitat de València, C. Dr. Moliner 50, 46100 Burjassot, València, Spain \\ 2 European Southern Observatory, Karl-Schwarzschild-Str. 2, 85748 Garching bei München, Germany \\ 3 Université Côte d'Azur, Observatoire de la Côte d'Azur, CNRS, Lagrange, CS 34229 Nice, France \\ 4 Department of Physics and Astronomy, Georgia State University, PO Box 5060 Atlanta, GA 30302-5060 \\ 5 Observatori Astronòmic, Universitat de València, Parc Científic, C. Catedrático José Beltrán 2, 46980 Paterna, València, Spain \\ 6 Theoretical Astrophysics, Department of Physics and Astronomy, Uppsala University, Box 516, SE-751 20 Uppsala, Sweden \\ 7 European Southern Observatory, Casilla 19001, Santiago 19, Chile
}

Received ...; accepted ...

\begin{abstract}
Context. Red supergiant stars possess surface features and extended molecular atmospheres. Photospheric convection may be a crucial factor of the levitation of the outer atmospheric layers. However, the mechanism responsible is still poorly understood.

Aims. We image the stellar surface of V602 Carinae (V602 Car) to constrain the morphology and contrast of the surface features and of the extended atmospheric layers.

Methods. We observed V602 Car with the Very Large Telescope Interferometer (VLTI) PIONIER instrument (1.53-1.78 $\mu \mathrm{m})$ between May and July 2016, and April and July 2019 with different telescope configurations. We compared the image reconstructions with 81 temporal snapshots of 3D radiative-hydrodynamics (RHD) $\mathrm{CO}^{5} \mathrm{BOLD}$ simulations in terms of contrast and morphology, using the Structural Similarity Index.

Results. The interferometric data are compatible with an overall spherical disk of angular diameter $4.4 \pm 0.2$ mas, and an extended molecular layer. In 2016, the reconstructed image reveals a bright arc-like feature toward the northern rim of the photospheric surface. In 2019, an arc-like feature is seen at a different orientation and a new peak of emission is detected on the opposite side. The contrasts of the reconstructed surface images are $11 \% \pm 2 \%$ and $9 \% \pm 2 \%$ for 2016 and 2019 , respectively. The morphology and contrast of the two images are consistent with 3D RHD simulations, within our achieved spatial resolution and dynamic range. The extended molecular layer contributes $10-13 \%$ of the total flux with an angular diameter of 6-8 mas. It is present but not clearly visible in the reconstructed images because it is close to the limits of the achieved dynamic range. The presence of the molecular layer is not reproduced by the 3D RHD simulations.

Conclusions. 3D RHD simulations predict substructures similar to the observed surface features of V602 Car at two different epochs. We interpret the structure on the stellar surface as being related to instationary convection. This structure is further convolved to larger observed patches on the stellar surface with our observational spatial resolution. Even though the simulations reproduce the observed features on the stellar surface, convection alone may not be the only relevant process that is levitating the atmosphere.
\end{abstract}

Key words. Techniques: image processing - Stars: atmospheres - Stars: imaging - Stars: late-type - Stars: massive - Stars: individual: V602 Car

\section{Introduction}

Red supergiants (RSGs) are cool evolved massive stars before their transition toward Wolf-Rayet (WR) stars and core-collapse supernovae. Their characterization and their observed location in the Hertzsprung-Russell (HR) diagram is important in order to calibrate stellar evolutionary models for massive stars and to understand their further evolution toward WR stars and supernovae (e.g., Dessart et al. 2013, Groh et al. 2013, 2014; Smith 2014; Meynet et al. 2015). Moreover, red supergiants are of importance in stellar synthesis models because of their high luminosities and high masses (e.g., Marco \& Negueruela 2013).

\footnotetext{
* Based on observations made with the Very Large Telescope Interferometer at the Paranal Observatory under program IDs 097.D-0286, 60.A-9138 (NAOMI science verification) and 2103.D-5029.

$\star \star$ Email: j.bautista.climent@uv.es

$\star \star \star$ Visiting Professor at the Department of Quantum Physics and Astrophysics, and the Institute of Cosmos Sciences of the University of Barcelona
}

The structure and morphology of the close circumstellar environment and wind regions, including the atmospheric molecular layers and dusty envelopes, are currently a matter of intense debate (e.g., Yoon \& Cantiello 2010, Walmswell \& Eldridge 2012). Knowledge of the circumstellar envelope and fundamental parameters is important to understand the matching of supernova (SN) progenitors to the different types of core-collapse $\mathrm{SNe}$ (Heger et al. 2003; Groh et al. 2013). The mass loss from red supergiants is, as well, one of the most important sources for the chemical enrichment of the interstellar medium.

The study of fundamental parameters and atmospheric extensions of RSGs in our neighborhood (Wittkowski et al. 2012, Arroyo-Torres et al. 2013, 2014, 2015; Wittkowski et al.|2017b) has shown that extended molecular atmospheres, with extensions comparable to Mira variable asymptotic giant branch (AGB) stars, are a common feature of RSGs stars and that, unlike for Miras, this phenomenon is not predicted by 3D radiativehydrodynamics (RHD) or 1D pulsation models (Arroyo-Torres et al. 2015). 
The onset of the mass-loss process, that is the levitation of the outer atmospheric layers to radii where dust can form, is currently not understood for RSG stars. The most commonly proposed mechanism has been an interplay of pulsation and convection (e.g., Yoon \& Cantiello 2010). Josselin \& Plez (2007) suggested that a decrease in the effective gravity, caused by convective motions, combined with radiative pressure on molecular lines, may initiate the mass loss in RSG stars. It was also suggested that magnetic fields could contribute to the heating of the outer atmosphere and to the mass loss (Aurière et al. 2010). Arroyo-Torres et al. (2015) showed that current 1D and $3 \mathrm{D}$ radiative-hydrodynamics models of pulsation and convection alone cannot levitate the molecular atmospheres of RSGs to observed extensions. They observed a correlation of atmospheric extension with luminosity, which may support a scenario that includes radiative acceleration on Doppler-shifted molecular lines. However, there are alternative mechanisms such as magnetic fields and Alfvén waves (e.g., Airapetian et al. 2010, Cranmer \& Saar 2011; Thirumalai \& Heyl| 2012; Rau et al.| 2019; Yasuda et al. 2019), differential rotation (Vlemmings et al. 2018), or the presence of giant dominating hot spots (recently observed by Montargès et al. 2016). Although the processes that initiate the mass loss from RSG stars are not currently known, it is well established that RSG stars show mass-loss rates between $2 \times$ $10^{-7} \mathrm{M}_{\odot} \mathrm{yr}^{-1}$ to $3 \times 10^{-4} \mathrm{M}_{\odot} \mathrm{yr}^{-1}$ (De Beck et al. 2010).

In this work our aim is to characterize the effects of convection on the stellar surface and to investigate the role that convection may play in the mass-loss process of RSGs. We compare VLTI-PIONIER image reconstructions of the stellar surface of V602 Carinae (V602 Car) with predictions by 3D simulations of stellar convection. We chose V602 Car as our main target, which was part of our previous sample of VLTI-AMBER studies, i.e., targets for which we had already established the fundamental stellar parameters and the presence of extended molecular atmospheres. Arroyo-Torres et al. (2015) reported for V602 Car a radius of $1050 \pm 165 \mathrm{R}_{\odot}$, an effective temperature of $3432 \pm 280 \mathrm{~K}$, a surface gravity $\log g=-0.30 \pm 0.16$, and an initial mass of 20$25 \mathrm{M}_{\odot}$ corresponding to a current mass of $10-13 \mathrm{M}_{\odot}$.

\section{Observations and data reduction}

We obtained interferometric observations of V602 Car employing the PIONIER instrument (Le Bouquin et al. 2011) of the Very Large Telescope Interferometer (VLTI) and its four auxiliary telescopes (ATs). The ATs were placed in three different effective configurations: short, medium, and long (see Table 1). Observations were taken using the ESO service mode between 7 April 2016 and 27 June 2016, and between 29 April 2019 and 8 July 2019. The 2019 data were taken using the new NAOMI adaptive optics system (Woillez et al. 2019) at the ATs during NAOMI science verification, providing an improved precision and accuracy compared to 2016 (see Appendix A for details). The data were dispersed over six spectral channels with central wavelengths $1.53 \mu \mathrm{m}, 1.58 \mu \mathrm{m}, 1.63 \mu \mathrm{m}, 1.68 \mu \mathrm{m}, 1.72 \mu \mathrm{m}$, $1.77 \mu \mathrm{m}$ and widths of $\sim 0.05 \mu \mathrm{m}$. Observations of V602 Car were interleaved with observations of the interferometric calibrator HD 96566 with spectral type G8III and an angular uniform disk diameter of $1.50 \pm 0.11$ mas (Lafrasse et al. 2010). A log of our observations can be found in Table 1 We initially divided the 2016 observation dates into three sub-epochs, where each epoch lasted not more than 9 days, because the V602 Car is a semi-regular variable. However, an analysis of the different sub-epochs showed, within our accuracy and spatial resolution, that there was no significant variability of the visibility data over the sub-epochs, so that in the following we analyzed the data of all sub-epochs together. We did not repeat the exact $u v$ coverage within the full epoch so that variability on small scales might be present and would be smeared by combining the data. The 3D convection models of RSGs by Chiavassa et al. (2009) showed time variations of surface structures on timescales of one month in the H-band. However, we show in Sect.5.2 that snapshots of convection simulations are similar at our spatial resolution, albeit not identical, on timescales of about 3 months, justifying our approach of combining the sub-epochs. The same reasoning was applied for the 2019 observations. The total $u v$ coverage that we obtained for our observations is very similar for 2016 and 2019, as can be seen in Fig. 1 .

We reduced and calibrated the data with the pndrs package (Le Bouquin et al. 2011). The resulting visibility data of our observations can be found in Fig. 2, together with model fits and synthetic visibilites of our image reconstructions, as discussed below.

\section{Data analysis}

The visibility data in Fig. 2 indicate an overall spherical stellar disk. However, deviations from a continuously decreasing visibility in the first lobe and closure phases different from $0 / 180^{\circ}$ at higher spatial frequencies indicate the presence of inhomogeneities.

As detected in previous K-band observations (Arroyo-Torres et al.2015), V602 Car possesses an extended molecular layer, in the near-IR most importantly of $\mathrm{H}_{2} \mathrm{O}$ and $\mathrm{CO}$, also called MOLsphere (Tsuji 2000). These same molecules are also present in the H-band, and such extended layers have been detected in the H-band, for example for the AGB star R Aquarii (Ragland et al. 2008). We should thus expect the MOLsphere of V602 Car to be seen in our H-band data as well. In order to describe the stellar photosphere and this MOLsphere, we used a two-component model: a PHOENIX model atmosphere (Hauschildt \& Baron 1999) for the stellar photosphere and a uniform disk (UD) de-

Table 1. Observation log of V602 Car with the instrument PIONIER.

\begin{tabular}{cccrr}
\hline \hline Date & Stations & Conf. $^{a}$ & $\begin{array}{r}\text { Seeing } \\
\left(^{\prime \prime}\right)\end{array}$ & $\begin{array}{r}\text { Coh. time } \\
(\mathrm{msec})\end{array}$ \\
\hline $2016-04-07$ & A0/G1/J2/J3 & $\mathrm{L}$ & 0.47 & 7.6 \\
$2016-05-23$ & A0/B2/C1/D0 & $\mathrm{S}$ & 0.63 & 4.4 \\
$2016-05-24$ & A0/B2/C1/D0 & $\mathrm{S}$ & 0.44 & 5.4 \\
$2016-05-25$ & A0/B2/C1/D0 & $\mathrm{S}$ & 0.47 & 3.3 \\
$2016-05-31$ & D0/G2/J3/K0 & $\mathrm{M}$ & 0.66 & 3.5 \\
$2016-06-01$ & A0/G1/J2/J3 & $\mathrm{L}$ & 0.66 & 2.5 \\
$2016-06-27$ & A0/B2/C1/D0 & $\mathrm{S}$ & 0.62 & 3.1 \\
\hline $2019-04-29$ & A0/D0/G1/J3 & $\mathrm{L}$ & 0.75 & 5.3 \\
$2019-05-02$ & A0/G1/J2/J3 & $\mathrm{L}$ & 0.57 & 6.7 \\
$2019-05-03$ & A0/G1/J2/J3 & $\mathrm{L}$ & 0.46 & 13.5 \\
$2019-05-04$ & A0/G1/J2/J3 & $\mathrm{L}$ & 0.52 & 4.9 \\
$2019-05-10$ & A0/B2/C1/D0 & $\mathrm{S}$ & 1.09 & 2.2 \\
$2019-05-30$ & A0/B2/C1/D0 & $\mathrm{S}$ & 0.77 & 2.5 \\
$2019-05-31$ & A0/B2/C1/D0 & $\mathrm{S}$ & 0.70 & 2.6 \\
$2019-07-07$ & A0/G2/J2/J3 & $\mathrm{L}$ & 0.47 & 6.3 \\
$2019-07-08$ & D0/G2/J3/K0 & $\mathrm{M}$ & 0.46 & 6.9 \\
\hline
\end{tabular}

Notes. ${ }^{a}$ Short configuration (S): AT stations A0/B2/C1/D0, ground baselines 10-40 m; Medium configuration (M): D0/G2/J3/K0, 40-100 $\mathrm{m}$; Long configuration (L): A0/G1/J2/J3, A0/G2/J2/J3, and $\mathrm{A} 0 / \mathrm{G} 2 / \mathrm{J} 2 / \mathrm{J} 3,60-140 \mathrm{~m}$. 

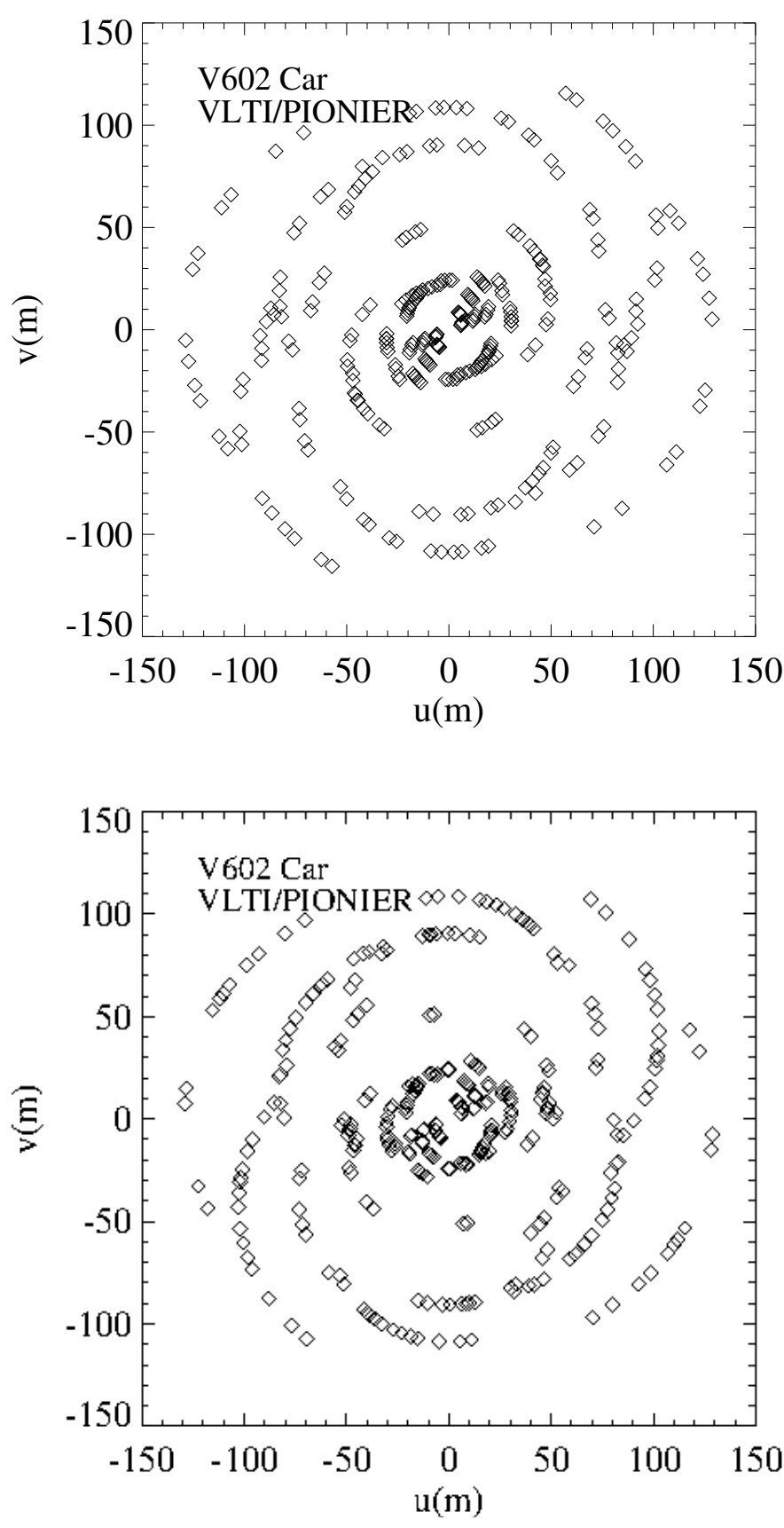

Fig. 1. Map of the $u v$ coverage of our PIONIER observations of V602 Car (upper, 2016; lower, 2019), where $u$ and $v$ are the spatial coordinates of the baselines projected on sky.

scribing the MOLsphere, as was done in Arroyo-Torres et al. (2015). We chose a PHOENIX model from the grid of ArroyoTorres et al. (2013) with parameters close to the established values for V602 Car by Arroyo-Torres et al. (2015): $20 \mathrm{M}_{\odot}, \mathrm{T}_{\mathrm{eff}}$ $=3400 \mathrm{~K}$, and $\log (\mathrm{g})=-0.5$. The fit was performed in the same way as in Wittkowski et al. (2017b) and separately for each spectral channel. We treated the flux fractions $f_{\text {Ross }}$ and $f_{\mathrm{UD}}$ as free parameters to allow for an additional over-resolved background component.

Table 2 lists the resulting best-fit parameters, together with the values averaged over the spectral channels. As expected for long-period variables, the flux contribution of the molecular layer is stronger in the water vapor bands toward the edges of the H-band. The angular diameter of the MOLsphere may not correlate well with its flux contribution, and may be less well constrained, in particular for low flux contributions. For the 2016 epoch, the best fit was found to be a photosphere with an angular diameter $\Theta_{\text {Ross }}$ of $4.4 \pm 0.2$ mas and a MOLsphere contributing on average $\sim 10 \%$ of the total flux with an angular diameter of $\sim 8$ mas. For the 2019 epoch, we obtained consistent values with a $\Theta_{\text {Ross }}$ of $4.5 \pm 0.2$ mas and a MOLsphere with the same parameters as for 2016. For both epochs the flux fraction of a larger unresolved component was negligible, and our values of the Rosseland angular diameter are consistent with the estimate of $\Theta_{\text {Ross }}=5.08 \pm 0.75$ mas by Arroyo-Torres et al. (2015).

The synthetic squared visibility values are plotted in Fig. 2 The PHOENIX plus MOLsphere model successfully describes our visibility data. The effect of the UD representing the MOLsphere is clearly visible, since the PHOENIX model alone is unable to reproduce the measured shape of the visibility function.

A close inspection of the visibilities at baselines 50-90 M $\lambda$ (Fig. 2), in particular for the 2016 epoch, reveals the presence of more than one visibility minimum along different baseline angles, where visibility minima are separated by about $5 \%$ in baseline length. As discussed for Betelgeuse (Chiavassa et al. 2010, Montargès et al. 2016), this feature may indicate that the star is seen by the interferometer as an overall slightly elongated disk, with differences of about 5\% in radius across different angles, instead of a perfectly spherical disk. However, as shown by Chiavassa et al. (2009, 2010), big intense convection cells within an overall spherical stellar disk can also be the origin of such dispersion of the spatial frequency at the visibility null. In order to probe this possibility, we reconstructed the observational images from these visibilities.

\section{Aperture synthesis imaging}

We used the reconstruction package SQUEEZE (Baron et al. 2010) to obtain aperture synthesis images.

We expect an overall (star plus stellar environment) source size beyond 8 mas in angular diameter based on the analysis in Sect. 3. As defined in Monnier (2003), the nominal spatial resolution of our imaging observations $\lambda /\left(2 B_{\max }\right)$ is 1.2 mas. We tested three different scenarios to select the best pixel size and field of view (FOV) for our image reconstructions: 1) 0.6 mas/pixel with $64 \times 64$ pixel FOV; 2) 0.3 mas/pixel with $64 \times 64$ pixel FOV; 3) 0.3 mas/pixel with $128 \times 128$ pixel FOV. When restricting the field of view (comparing scenarios 2 and 3 ), the $\chi^{2}$ of the reconstructed images clearly favored scenario 3 , that with a larger FOV. This is a good indication of extended flux that scenario 2 is not able to recover. The visual comparison of scenarios 1 and 3 (i.e., the same FOV with different resolutions) showed the same features in both images. No new information was created with the pixel size of 0.3 mas/pixel, but $\chi^{2}$ improved, so we kept this as our final pixel size and FOV.

We performed SQUEEZE reconstructions for two main regularizations: Laplacian $(l a)$ and Total Variation $(t v)$. For both of these regularizations we also included an L0-norm regularization $(l 0)$ to decrease the number of spurious point-like sources in the FOV. We also used a transpectral regularization $(t s)$ to center all the images in the bandpass at the same position of the FOV (when working with combined channels). We tested two combinations of regularizations: $l a+l 0+t s$ and $t v+l 0+t s$. The optimum value of a given regularization's hyperparameter $(\mu)$ was selected in the following way. We created an L-curve characterizing the response of the prior term versus the $\chi^{2}$ value of the image solution for several values of $\mu$. The optimum value of 

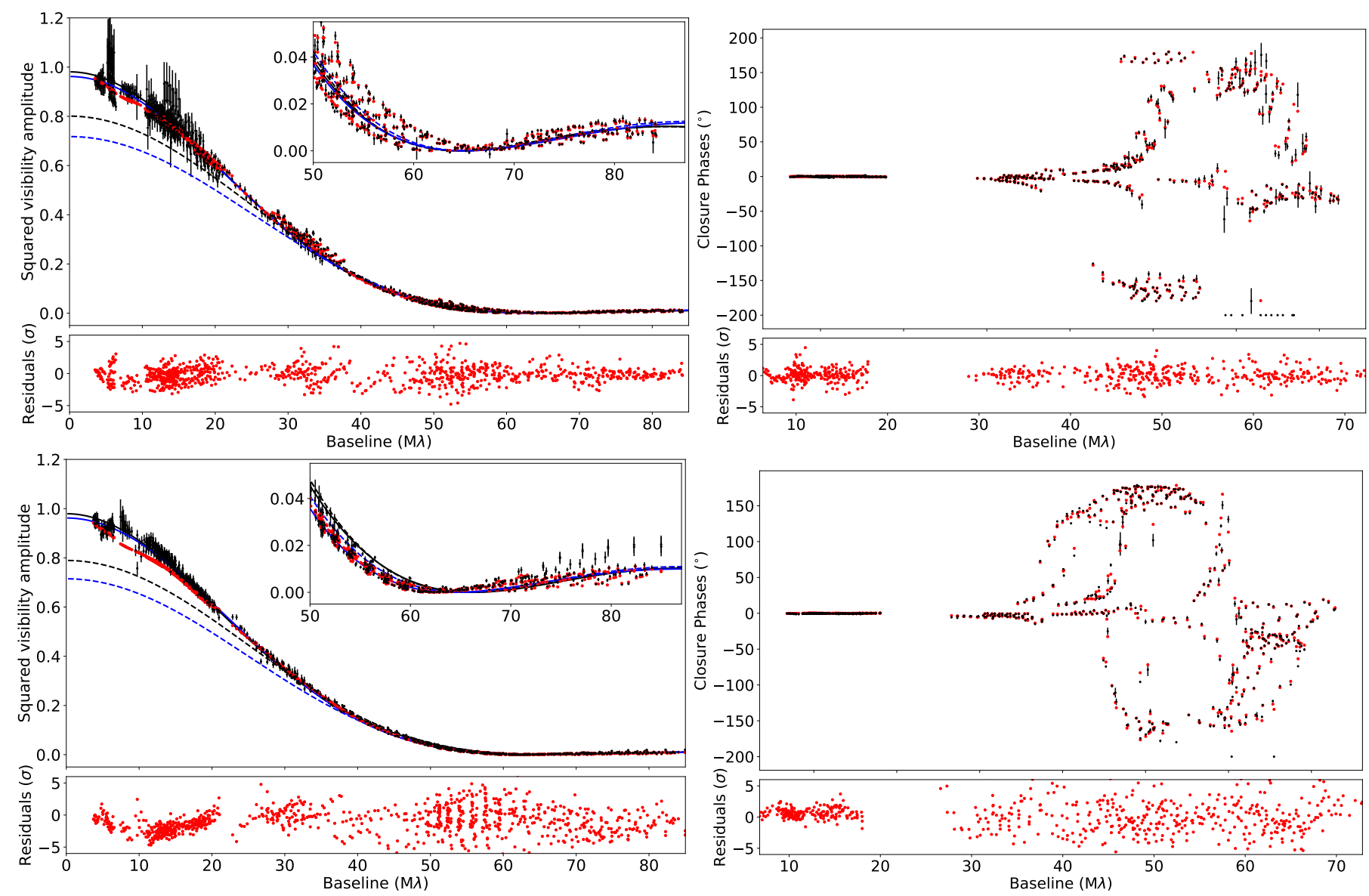

Fig. 2. PIONIER visibility results of V602 Car of 2016 (top) and 2019 (bottom) as a function of baseline length. The left panel shows the squared visibility amplitudes, where the inlay enlarges the part of the low values. The right panel shows the closure phases. The vertical bars indicate the symmetric error bars. The black solid line denotes our visibility model including the stellar photosphere, represented by a PHOENIX model atmosphere, and a larger uniform disk indicating the extended atmosphere or MOLsphere. The black dashed line indicates the part of the PHOENIX model atmosphere alone without the added uniform disk (MOLsphere). The blue solid and dashed lines represent the same parts, but for the selected 3D RHD snapshots for each epoch instead of the PHOENIX model (see Sect. 5. The synthetic values based on the reconstructed images are shown in red (SQUEEZE algorithm). The small panels below the main panels provide the residuals between observations and reconstructed images.
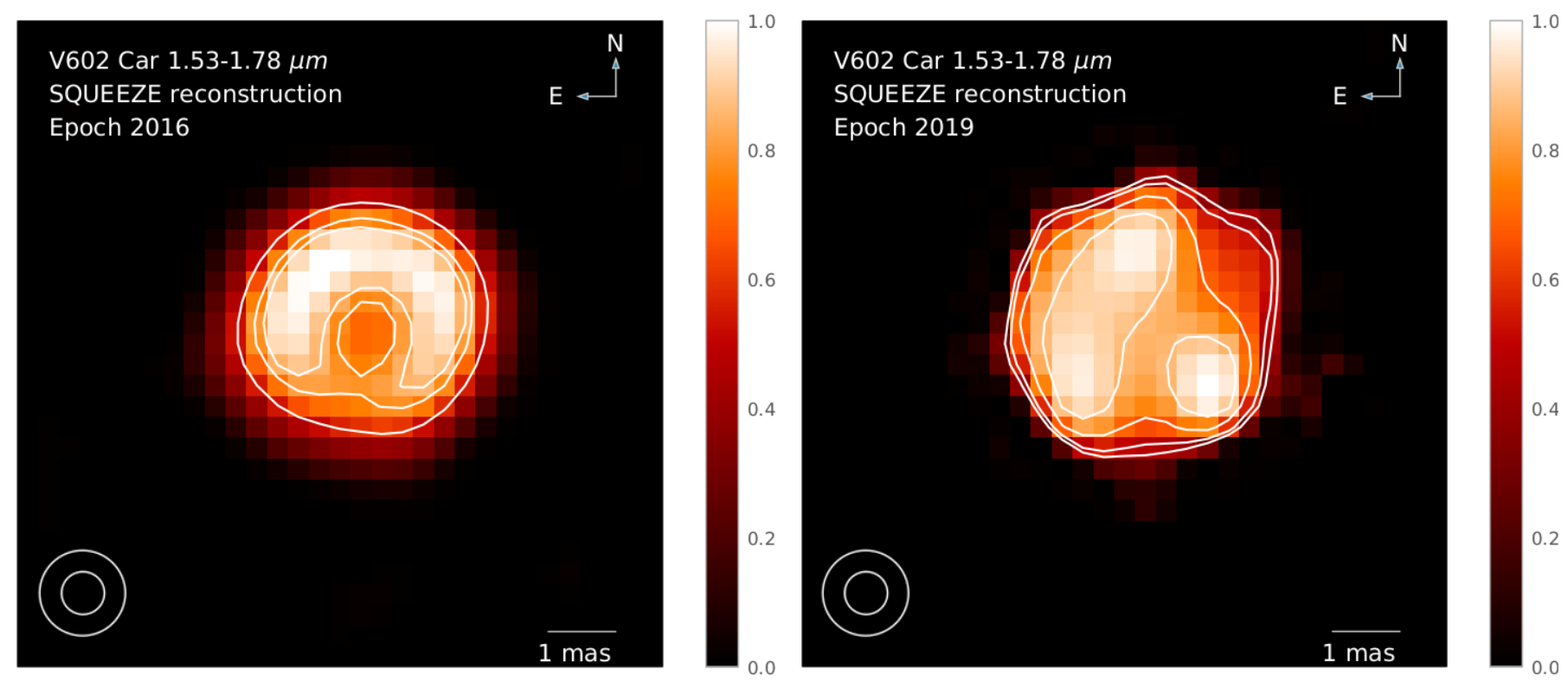

Fig. 3. SQUEEZE reconstruction of V602 Car with spectral channels combined. (Left): 2016 data set. Contours are drawn at levels 55\%, 77\% and $85 \%$ of the peak intensity. (Right). 2019 data set. In this case, contours are drawn at levels $40 \%, 50 \%, 70 \%$, and $87 \%$ of the peak intensity. Here and hereafter, the pixel scale is 0.3 mas/pixel. The size of the circles in the lower left corners indicate our nominal angular resolution of 1.2 mas and the smallest circle represents our best estimate of the real resolution obtained, 0.6 mas (see Appendix B.3. 
$\mu$ is associated with the elbow of the L-curve. This procedure ${ }^{1}$ was followed first for the $l 0$ regularization, then for the $t s$ regularization, and finally for the $l a$ and $t v$ regularizations.

We also tested the possible influence of an initial model on the image reconstruction process. The image reconstructions without an initial model were obtained by employing the procedure explained in Paladini et al. (2018); the steps are as follows: i) create a reconstructed image with a resolution of one-quarter the number of pixels and four times the mas/pixel of the final image, with a simple Dirac delta function as a start image; ii) use this image as the initial guess for creating another one with onehalf the number of pixels and two times the mas/pixel of the final image; iii) using this intermediate image as initial model, reconstruct the final image at full resolution. The reconstructions with initial model used the best-fit models from the PHOENIX + UD model discussed in Sect. 3. The difference between these two methods of reconstruction (initial model versus no initial model) was negligible.

We first reconstructed images at the six spectral channels individually. When comparing the images, we did not find significant differences across spectral channels. The structural similarity index (SSIM; see Sect. 5.2) showed a very high similarity (0.99) across the spectral channels. Therefore, we combined the data of all spectral channels covering wavelengths of $1.53 \mu \mathrm{m}$ to $1.78 \mu \mathrm{m}$.

We selected the SQUEEZE images with lowest $\chi^{2}$; in the case of combined channels for 2016 this corresponds to $\chi^{2}=$ 1.57 with $\mu_{t v}=500, \mu_{l 0}=3, \mu_{t s}=1$, using the initial model described in Sect. 3. The 2019 combined channels image has a $\chi^{2}$ $=7.09$ with $\mu_{t v}=2000, \mu_{l 0}=30, \mu_{t s}=1$, also using an initial model. The reason of the larger $\chi^{2}$ of the 2019 image compared to the 2016 image is not clear. It may be related to the smaller estimated errors of the measured visibility and closure phases in the 2019 data, so that systematic absolute calibration uncertainties have a larger relative contribution. Our image reconstruction tests (see Sect. 4.2) confirm that the 2019 image reconstruction is as at least as reliable as the 2016 image reconstruction. The reconstructed images were not further convolved beyond the chosen pixel scale of $0.3 \mathrm{mas} / \mathrm{pixel}$, as discussed above.

\subsection{Final reconstructed images}

Figure 3 shows the final reconstructed images for the 2016 and 2019 epochs. The images of the individual spectral channels are shown in Appendix C to illustrate that they are very similar across all the spectral channels. We obtained the synthetic visibilities of the final reconstructed images at our $u v$ observational points using the OITOOLS package The comparison of the interferometric observables from the experimental data with those extracted from the reconstructed images (Fig. 3) shows a very good agreement (Fig. 2). This confirms that extended flux caused by the MOLsphere (Sect. 3) is present in the reconstructed images, as already indicated by the improved $\chi^{2}$ values with increased FOV (Sect. 4). For the sake of clarity, we show in Fig. 2 only the visibility values based on the wavelengthand time-averaged reconstructions, while the observed visibilities are shown for individual observing dates and spectral channels. Some of the residual differences in Fig. 2 may be caused by this effect.

\footnotetext{
${ }_{1}$ See Reconstruction test report and data processing cookbooks by Sanchez-Bermudez et al., available at http://www.jmmc.fr/ oimaging.htm

${ }_{2}$ Available at https://github.com/fabienbaron/OITOOLS.jl
}

The reconstructed image of the 2016 epoch shows the stellar disk with an intriguing, bright arc-like feature toward the northern rim of the stellar surface. In 2019, the orientation of the arclike feature is different and a new peak of emission is detected on the opposite side of the stellar surface. The extended molecular layer or MOLsphere, although present in the reconstructed images, lies close to our achieved dynamic range (of about 1:10 to $1: 20$ ), so that it is not clearly visible. While the parameters of the MOLsphere show a dependence on wavelength, the photospheric structure is not expected to be wavelength dependent, which explains why the reconstructed images appear to be very similar across spectral channels.

The double visibility null, as seen in the observed visibilities in Fig. 2 and described in Sect. 3 , is reproduced by the image reconstructions, and is thus most likely caused by the surface features and not by an overall elongated stellar disk.

\subsection{Error estimates of the final reconstructed image}

We characterized possible errors that may be introduced by the reconstruction process to assess the soundness of the detected surface features. We also used the IRBis reconstruction package (Hofmann et al. 2014, Image Reconstruction software using the Bispectrum) to test the dependency of our results on the reconstruction package employed. A detailed explanation of these tests can be found in Appendix B. Our analysis revealed the following: i) No new features are introduced within SQUEEZE when altering the final reconstructed images by one standard deviation; ii) Synthetic observational data based on 3D snapshots at our $u v$ points and with our level of noise recovers the substructure present in the original image, with maximum intensity losses of $26 \%$ for the 2016 and $30 \%$ for the 2019 epochs; iii) The difference between SQUEEZE and IRBis reconstructed images shows that the same structures are present in both image reconstructions. Therefore, we conclude that the detected structure is most likely real and not due to any artificial effect.

Figure 4 shows the total error map, conservatively taking into account all these possible error sources, as described in detail in Appendix $B$ The average errors, in terms of original image flux, are $17 \%$ and $14 \%$ for 2016 and 2019, respectively. Most of these error sources are systematic, extending across the images, so that the pixel-to-pixel error is significantly smaller.

Our tests using reconstructions of synthetic data (based on 3D RHD models and with our $u v$ coverage and observational errors) with different convolution kernels (see Appendix B.3 revealed that original images and reconstructions match best with a convolution kernel of 0.6 mas. This suggests that we reach with our data and $u v$ coverage a super-resolution of $\sim 0.6$ mas compared to the nominal resolution $\lambda /\left(2 B_{\max }\right)$ of 1.2 mas.

\section{Comparison with 3D RHD simulations}

In order to compare our PIONIER data of V602 Car to theoretical models, we used numerical 3D RHD simulations obtained with the $\mathrm{CO}^{5} \mathrm{BOLD}$ code (COnservative COde for the COmputation of COmpressible COnvection in a BOx of L Dimensions; Freytag et al. 2012). The simulation used was st35gm04n38 $\left(401^{3}\right.$ grid points, $\mathrm{T}_{\mathrm{eff}}=3414 \pm 17 \mathrm{~K}, \log g=-0.39 \pm 0.01,5 \mathrm{M}_{\odot}$, $582 \pm 5 \mathrm{R}_{\odot}$ ). The grid resolution is $4.055 \mathrm{R}_{\odot}$ with a total field of view of $1626 \mathrm{R}_{\odot}$. This model shows an effective temperature and surface gravity as established for V602 Car (see Sect. 1), while it has a smaller radius and a lower mass compared to the observational parameters. Due to the limited number of currently avail- 

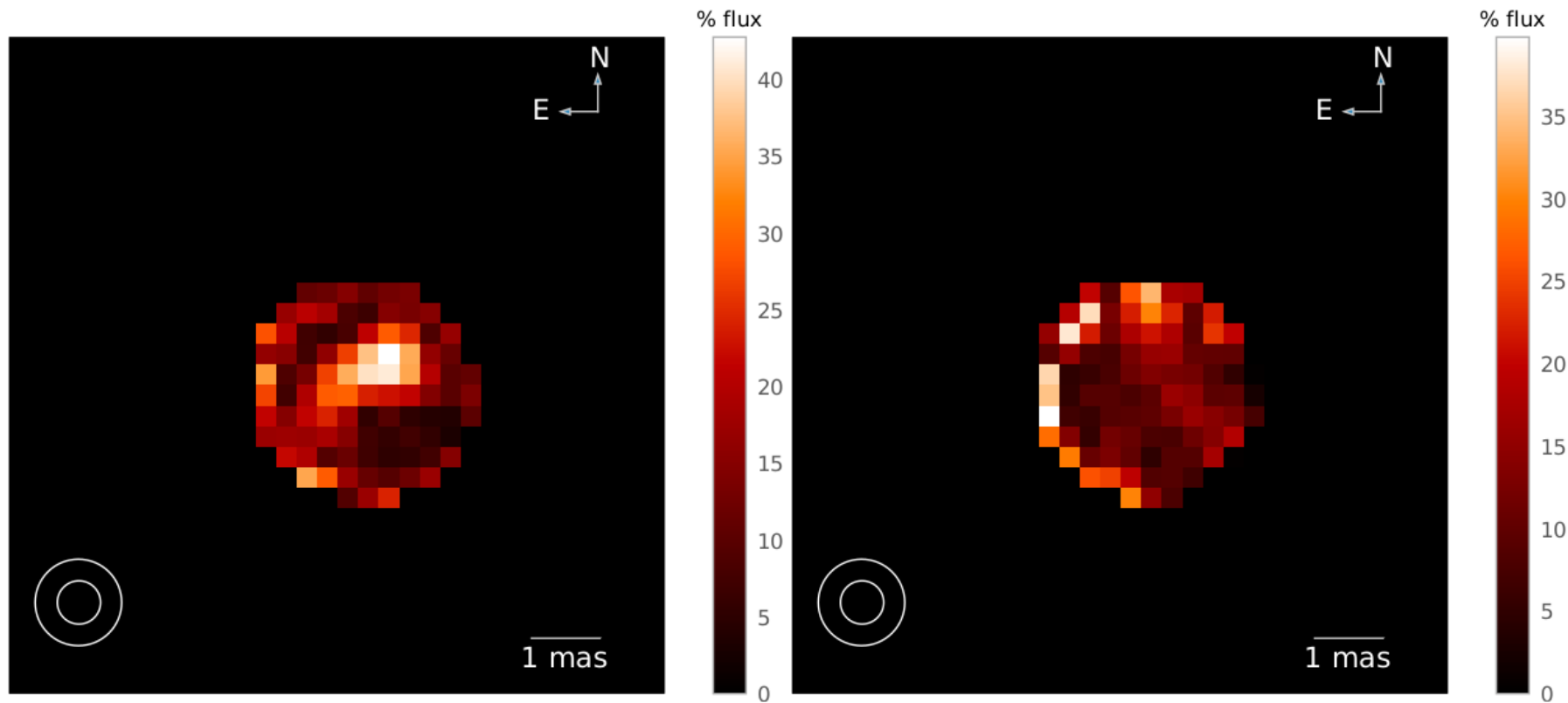

Fig. 4. Error images for the 2016 epoch (left) and 2019 epoch (right) resulting conservatively from the addition of three possible sources of errors discussed in Appendix B The errors are expressed in terms of the original image flux. Here and hereafter, the 3D-RHD-model-related images have being convolved with a 0.6 mas beam, that is, our best estimate of the real resolution obtained (see Appendix B.3.

able 3D simulations of RSG stars, and in particular to the computationally demanding calculation of higher mass stellar models, 3D models of a current mass of $10-13 \mathrm{M}_{\odot}$, as expected for $\mathrm{V} 602$ Car, and larger radii are not yet available. Nevertheless, this 3D model represents typical properties of an RSG star, and distinctively different dynamical properties than lower-mass pulsating AGB star models (cf. the discussion by Kravchenko et al. 2019). This simulation reproduces the effects of convection and, additionally, non-radial waves (Chiavassa et al. 2011). This model was first used by Kravchenko et al. (2018) and Kravchenko et al. (2019), and a detailed discussion on the model can be found therein. We computed 81 temporal snapshots about 23 days apart and covering a stellar time of about 1863 days in total. Intensity images were then computed using the pure-LTE radiative transfer Optim3D (Chiavassa et al.2009) at the bandpass of our PIONIER observation of $1.65 \pm 0.15 \mu \mathrm{m}$ (averaged over 56 maps across the range $1.5-1.8 \mu \mathrm{m})$.

\subsection{Comparison in terms of contrast}

We estimated the contrast of our reconstructed images, $\delta I_{r m s} /\langle I\rangle$, as defined in Tremblay et al. (2013) to compare them to 3D RHD simulations. The contrast on the stellar surface is affected both by surface features and by the limb-darkening (LD) effect. We are interested in the contrast of the surface features themselves. In order to correct for the LD effect, we used two independent methods: i) dividing the reconstructed image by the best-fit model image described in Sect. 3, ii) applying Equation 2 found in Chiavassa et al. (2009) with parameters in Table 2 of the same text. In this second method we created a high resolution image of the LD model $(401 \times 401$ pixels) with the same field of view (FOV) as the reconstructed image. Then we re-binned it to the same resolution and pixel size of our reconstucted image, so that both images (LD model and reconstructed) possessed the same FOV and resolution. Finally, we divided pixel by pixel in a similar fashion to the first method. Both of these methods resulted in a very similar correction. From now on the results exposed are valid for both of them.
We need to ensure that we do not include the stellar limb in our estimate of the contrast of surface features. We define a cutoff radius, i.e., the maximum radius adopted from the center of the star and for all angles, from which the outer pixels are not considered to compute the contrast. Figure 5 shows the contrast as a function of the chosen cut-off radius. For the 2016 data, the contrast increases with increasing radial cut up to $\sim 0.5$ stellar radii. This could be explained by an increasing number of image patterns included as the cut-off radius gets larger. For larger cutoff radii, between about 0.75 and 0.95 stellar radii, the contrast again shows a fast increase. This may be an effect of the limbdarkening that may not be perfectly corrected. When the radial cut surpasses the value of about 0.95 stellar radii we see a rapid and steep increase, which may be representative of the contrast between the stellar disk and the outside of the disk, which may also not be perfectly circular. A similar behavior is seen for the 2019 data, where more patterns and structures are included as the radial cut gets larger, until a rapid increase occurs at $\sim 0.85$ stellar radii.

We only consider the contrast below a radial cut of 0.75 stellar radii to avoid the bias of the uncertainty of the limbdarkening correction. The lower cut must include the arc-like feature present in Fig. 3 for 2016 and the more complex features of the 2019 data. Therefore, we establish a lower cut of 0.5 stellar radii in the 2016 data, implying a contrast value of $11 \% \pm 2 \%$, which corresponds to the plateau found between these radii. Due to shape of the substructures present in 2019 data, no lower cut value can be easily determined for this epoch. Therefore, we assume as a radial cut the maximum value considered here (i.e., 0.75 stellar radii, with a contrast value of $9 \% \pm 2 \%$ ). Although the average pixel errors of the images were $17 \%$ and $14 \%$ for 2016 and 2019, as described in Sect. 4.1, and thus larger than the surface feature contrasts, we note that the pixel errors are conservative values that take into account multiple sources of errors, as outlined in Appendix B, several of which are systematic. This does not mean that the pixel-to-pixel uncertainties and the contrast uncertainties are as large as this error map. 

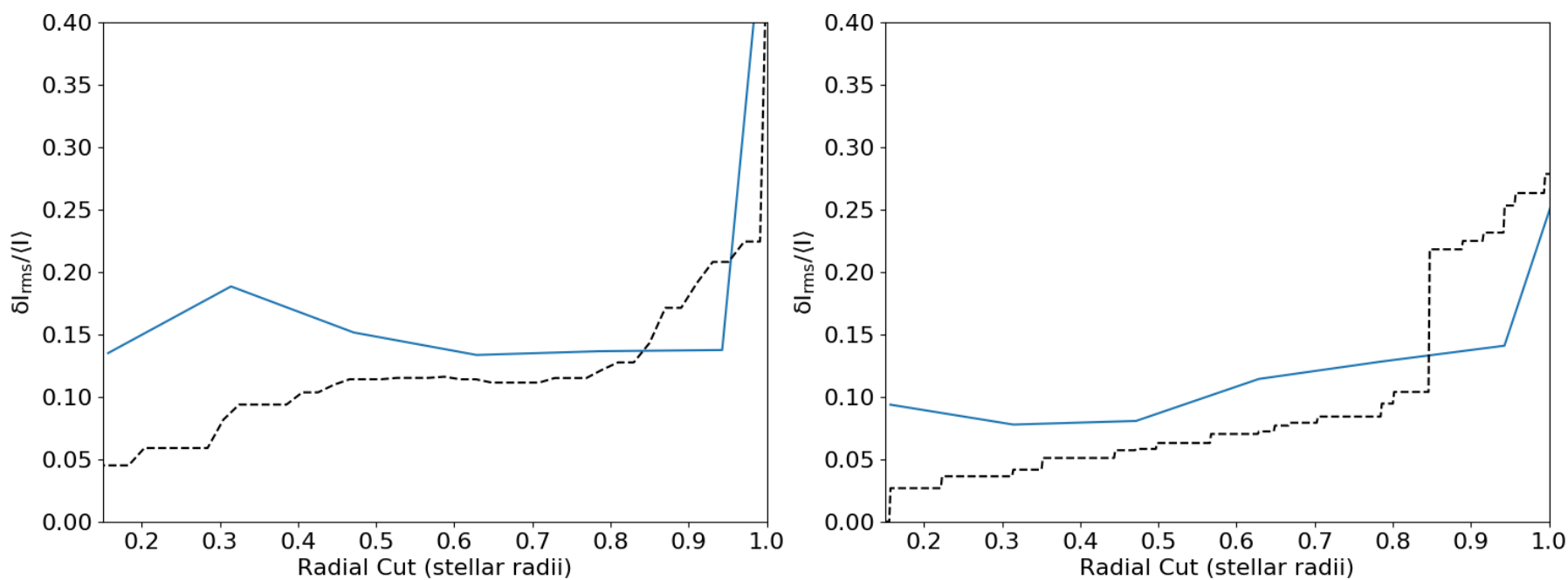

Fig. 5. Contrast of the data set as a function of the radial cut considered. The black dashed line indicates the contrast of the SQUEEZE reconstructed image (2016 in the left panel and 2019 in the right panel) and the blue solid line is one of the best simulated snapshot for each data set (snapshot 65 for 2016 data and snapshot 67 for 2019 data).

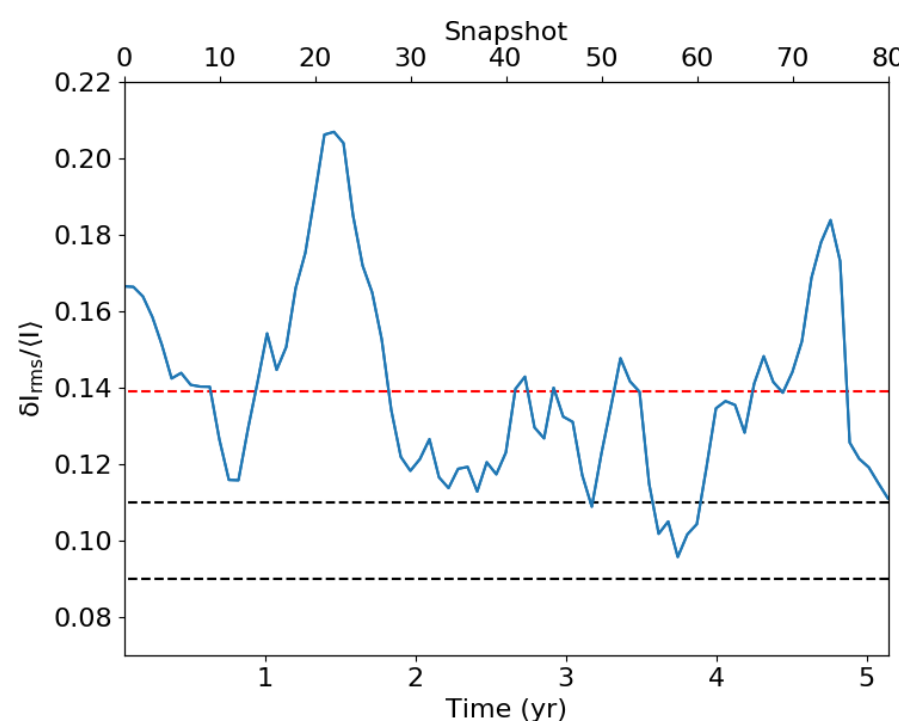

Fig. 6. The blue line represents the contrast of the simulated snapshots after being corrected for limb-darkening (LD) with a radial cut of 0.75 stellar radii. The black dashed lines represent the contrasts measured in the final reconstructed images ( $11 \pm 2 \%$ in 2016, $9 \pm 2 \%$ in 2019), while the orange dashed line represents the average value over all the snapshots $(14 \pm 2 \%)$. The LD was corrected using the best-fit model image from Sect. 3

Following the same procedure, we calculated the contrast for the 81 snapshots of the simulated 3D RHD snapshots. Figure 6 shows how the contrast varies across different snapshots, assuming a radial cut of 0.75 stellar radii. The average contrast value over the 81 snapshots is $14 \% \pm 2 \%$. This value is slightly larger than that of our image reconstructions, but with individual snapshots that have consistent contrast values.

Considering that our observational epochs lasted about 70 days, we made a test in which we first averaged consecutive model snapshots over this time span (2-3 snapshots) and then computed the contrasts of the averaged snapshots. The snapshot images were similar over these time spans, resulting in only marginal differences in the contrast curve shown in Fig. 6 .
Previous works have found similar contrast values in RSGs. Wittkowski et al. (2017a) consistently reported a contrast of $10 \% \pm 4 \%$ for the RSG V766 Cen, while Montargès et al. (2018) found a lower contrast of $5-6 \% \pm 1 \%$ for the RSG CE Tau. Both estimates were based on similar imaging of data obtained with the PIONIER instrument in the near-IR H-band.

\subsection{Comparison in terms of morphology}

We then investigated whether the 3D RHD simulations could reproduce the observed morphology of our reconstructed images, such as the arc-like feature discussed in Sect. 4.1.

The calculated snapshots of the 3D RHD simulations represent the stellar convection dynamics every $\sim 23$ days, covering a total of 1863 days. There is a fundamental problem in direct comparisons of such simulated snapshots to our data: the surface pattern changes in a stochastic way and never repeats itself. With a finite number of simulated snapshots, we cannot expect any snapshot to coincide exactly with the pattern at one of our observed epochs. If we cannot expect a model to describe the observational data, a formal $\chi^{2}$ comparison between model and observation is not appropriate and may lead to spurious results. As a solution to this fundamental problem, we introduce the use of the SSIM (Wang et al. 2004) to find the most similar of the 81 model snapshots compared to our reconstructed image (Fig. 3). The SSIM of a pair of images represents a superior method for image comparison. It is typically used in order to quantify the differences between a distorted image and a reference image. It is based on the perceived change in the structural information from one image to the other, and ranges from -1 to 1 , where 1 indicates perfect similarity (Eq. D.1).

We first convolved the model images to our best estimate of the real resolution obtained, 0.6 mas (see Appendix B.3, resized the images to the pixel scale and field of view of our reconstructed images, and applied a cut-off radius of 0.75 stellar radii as in Sect. 5.1. To account for the unknown orientation on the plane of the sky, we rotated the model images every $5^{\circ}$ around its center and estimated the SSIM for each rotation angle. As expected, none of the snapshots coincided with our observed epochs perfectly, but several were equally similar. For the 2016 data, the most similar snapshots were (rotation angle in parenthesis): $003\left(325^{\circ} \pm 5^{\circ}\right), 004\left(325^{\circ} \pm 5^{\circ}\right), 005\left(320^{\circ} \pm 5^{\circ}\right), 006$ 

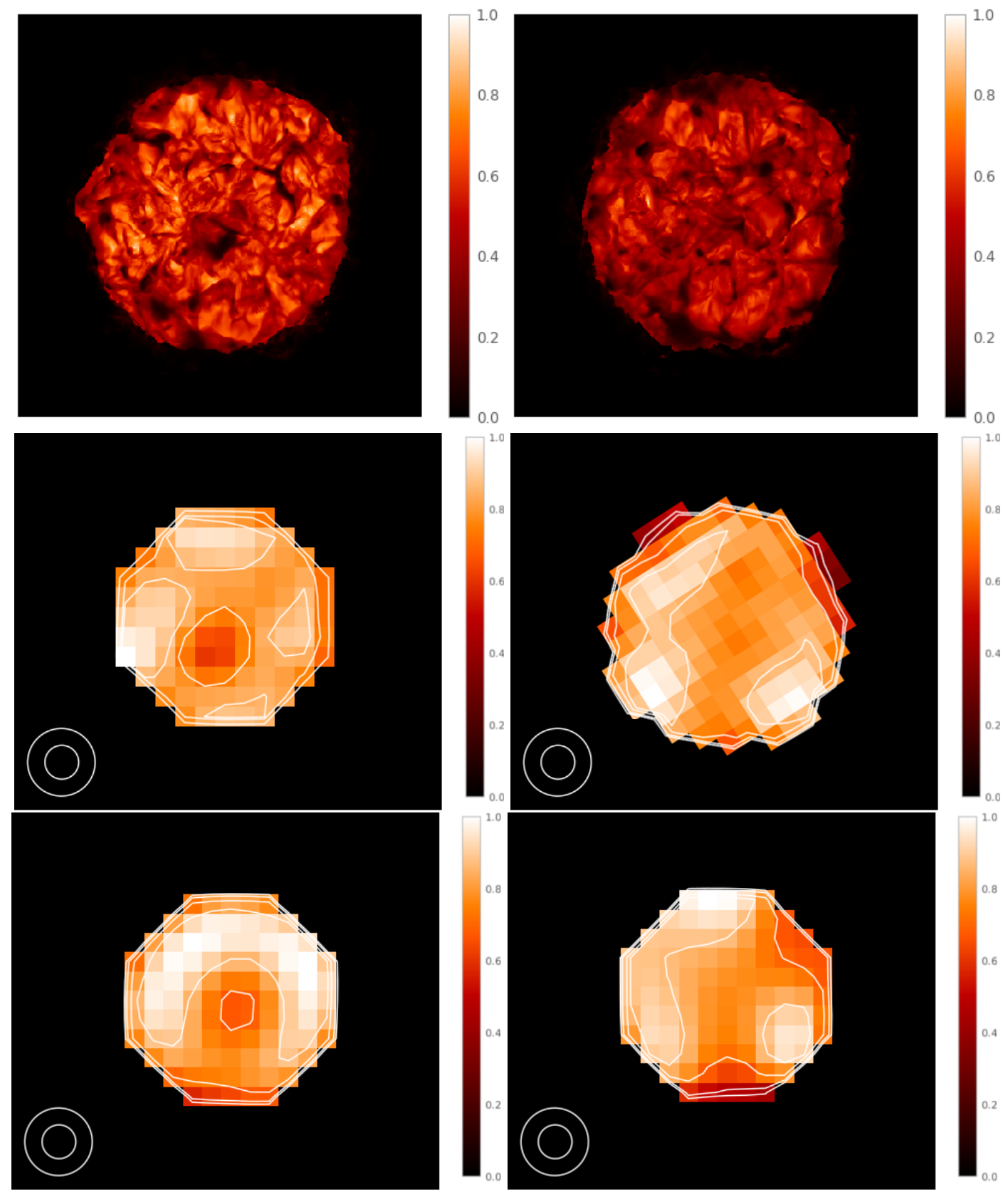

Fig. 7. Comparison in terms of morphology between model snapshots and reconstructed images. (Top row): Intensity image of one of the selected best snapshots (number 065) for 2016 in relative intensity (left), and for snapshot 067, which is the best choice for the 2019 data (right). (Middle row): The same snapshot images as in the upper row after being convolved with a 0.6 mas beam, rotated to match the observed morphology, and corrected for the limb-darkening effect with a cut-off radius of 0.75 stellar radii. (Bottom row): Reconstructed observational images after LD correction and with a cut-off radius of 0.75 stellar radii. In the middle and lower rows, the contours are drawn at levels of 55\%, 77\%, and $85 \%$ of the peak intensity for 2016 (left column) and at 40\%, 50\%, 70\%, and 87\% of the peak intensity for 2019 (right column).

$\left(325^{\circ} \pm 5^{\circ}\right), 064\left(10^{\circ} \pm 5^{\circ}\right), 065\left(10^{\circ} \pm 5^{\circ}\right), 066\left(10^{\circ} \pm 5^{\circ}\right)$, and $067\left(10^{\circ} \pm 5^{\circ}\right)$, all with SSIM $=0.85$. All of these snapshots coincided with those that we had previously selected to be most similar to our observed image (via visual inspection). We selected snapshot 065 as a representative of this subset of snapshots, in which the arc-like structure is visually clearest. Figure 7 (upper left panel) shows this model image at the original model resolution. Figure 7(left middle and lower panels) shows a comparison of this model image to our image reconstruction after adjusting it to the pixel size of the reconstruction, and rotating it to best match the reconstruction.
The same procedure was followed for the 2019 data selecting, in this case, snapshots $067\left(-30^{\circ} \pm 5^{\circ}\right), 068\left(-30^{\circ} \pm 5^{\circ}\right), 069$ $\left(-30^{\circ} \pm 5^{\circ}\right)$, and $070\left(-35^{\circ} \pm 5^{\circ}\right)$, all with SSIM $=0.87$. As a representative of this subset of snapshots, we selected snapshot 067 (see Fig. 7 right panels). Adjacent snapshots for the 2016 and 2019 data are very similar to these selected representatives. Although visually not identical to each other, our limited spatial resolution and dynamic range render them equally similar to the observational image. Finally, we tested the uncertainties when computing the SSIM by adding and subtracting the intensity error image (Fig. 4) to the observational images (Fig. 3) and computing the SSIM between these resulting images and the se- 
lected 3D RHD model images corresponding to each epoch. The SSIM value differs only 0.02 with respect to the case when no error image is considered.

The surface features seen in the model snapshots (Fig. 7 top row) are unlikely individual deep convection cells that reach out to the surface layers, as the timescale on which the structure changes in the models is too fast. The features in the model snapshots are therefore likely related to instationary convection, i.e., to pressure fluctuations (non-radial waves that do not exist long enough to produce a clear mode visible in a power spectrum) that are caused by sonic convective motions and are able to affect a single surface granule and also a group of neighboring granules. With the limited spatial resolution of our observation (Fig. 7, middle and bottom row), the structure is further convolved to larger observed patches on the stellar surface. This means that we cannot determine the sizes of individual granules or convection cells (see also Freytag 2003).

Each subset of the most similar snapshots to our 2016 data, 003 to 006 and 064 to 067 , represents a time span of 69.5 days, indicating that the structure remains similar on timescales of $\sim 2$ months. A similar result is found in the 2019 analysis where snapshots 067 to 070 also represent 69.5 days. This confirms, with our accuracy and spatial resolution, that it was a valid approach to combine data obtained over about 70 days (see Sect. 2).

The agreement between our image reconstructions and the most similar snapshots of the 3D RHD simulations may indicate, within our angular resolution and achieved dynamic range, that the observed stellar surface features of V602 Car at two individual epochs can be reproduced by the physics accounted for in the simulations we considered (i.e., non-local radiation transport, shock waves, gray and non-gray opacities; see Chiavassa et al.2011).

We then computed azimuthally averaged intensity profiles and synthetic visibility values for the selected snapshots and compared them to our observed visibility spectra. We computed the intensity profiles using rings regularly spaced in $\mu$, related to the impact parameter by $r / R_{\text {star }}=\sqrt{1-\mu^{2}}$. We used 56 spectral maps between $1.5 \mu \mathrm{m}$ and $1.8 \mu \mathrm{m}$ at a spectral resolution of 300 . Synthetic visibility values were then derived following the same procedure as in Wittkowski et al. (2017b), and in the same way as for the fit of a PHOENIX model in Sect. 3 Table 2 shows the resulting best-fit parameters. The synthetic visibilities based on this model are included in Fig. 2. In both the 2016 and 2019 data, the best-fit parameters are close to those obtained from the fit of the PHOENIX model together with a UD representing the MOLsphere. We adopted a resulting photospheric angular diameter of V602 Car of $4.4 \pm 0.2$ mas. This is the average of the photospheric angular diameters for 2016 and 2019, and are based on the fits including the PHOENIX model.

The best fits were achieved with an additional UD component and a free zero visibility scale, as for the 1D PHOENIX model atmosphere. The visibility plot of the best model for each epoch is included in Fig. 2. This shows that the 3D RHD simulations alone cannot reproduce the observed visibility values, but that an additional, more extended component is still required to reproduce the observed data. The presence of a MOLsphere on top of the photosphere may alter the contrast and morphology of the photospheric features to some extent (below about $4 \%$ in pixel value). This may explain a part of the residual differences between image reconstructions and 3D model images in terms of contrast and morphology.

\section{Conclusions}

Our new VLTI/PIONIER visibility data sets of V602 Car indicate an overall spherical stellar disk and an extended molecular layer, similarly to what has been detected in previous observations. The same data also indicate the presence of substructures within the stellar disk at the epochs 2016 and 2019. In order to further probe the stellar surface of V602 Car we obtained aperture synthesis images using two different reconstruction packages: SQUEEZE and IRBis. Both packages resulted in very similar results. The reconstructed images revealed a bright arc-like feature toward the northern rim of the stellar surface of the RSG V602 Car in 2016. Three years later, in 2019, an arc-like feature appeared at a different orientation and a new peak of emission emerged on the opposite side of the stellar surface. The flux contribution caused by the extended molecular layer is present in the reconstructed images, but it is not clearly visible because it lies close to our achieved dynamic range. We can therefore not constrain its morphology.

We compared the reconstructed images to the latest 3D RHD simulations of RSGs. There is a fundamental problem in direct comparisons of such simulated snapshots to our data: the surface pattern changes in a stochastic way and never repeats itself. With a finite number of available simulated snapshots, we cannot expect any snapshot to coincide exactly with the pattern at one of our observed epochs. A classic $\chi^{2}$ comparison between model and observation is thus not appropriate and may lead to spurious results. As a solution to this problem, we introduced the use of the SSIM to find the most similar of the model snapshots compared to our reconstructed image. This comparison resulted in the identification of 8 and 4 adjacent snapshots (out of 81 total) that are equally similar to the observational data obtained in 2016 and 2019, respectively. The SSIM was 0.85 and 0.87 , respectively, indicating that none of the snapshots coincides perfectly with our observed epochs, but some show a high degree of similarity. We concluded that, within our limitations in angular resolution and dynamic range, the observed stellar surface features of V602 Car can be reproduced by the physics accounted for in the simulations we considered at two individual epochs. Further observations at higher spatial and temporal resolution are needed to confirm the agreement. We interpreted the observed surface features to be related to instationary convection. The structure is further convolved to larger observed patches on the stellar surface with our observational spatial resolution. As a result, we lose information on the sizes of individual granules or convection cells. The time during which the structure of the most similar snapshots remains stable is $\sim 70$ days. As a more quantitative method of comparing observational data and simulations, we computed the contrast of the best snapshots and found agreement with the contrast of the reconstructed images (within the associated errors).

Although the observed stellar surface structure can be nicely explained by the 3D RHD models, the simulations alone are not able to reproduce the observed visibility data. An additional extended molecular component is still needed, pointing to the current limitations of RHD simulations of RSG stars, as found in Arroyo-Torres et al. (2015). While the effects of convection on the stellar surface may be nicely described by current 3D simulations and the physics they contain, convection alone may not be the only relevant process to levitate the atmosphere, which is the first step of the mass-loss process.

Acknowledgements. J.B.C., J.M.M, and J.C.G. were partially supported by the Spanish MINECO projects AYA2012-38491-C02-01 and AYA2015-63939C2-2-P and by the Generalitat Valenciana project PROMETEO/2009/104 and 
Table 2. Fit parameters to the PHOENIX model and to the best snapshots from 3D RHD simulations, for each epoch.

\begin{tabular}{|c|c|c|c|c|c|c|c|}
\hline Model & Epoch & Channel $(\mu m)$ & $\Theta_{1}$ (mas) & $f_{1}(\%)$ & $\Theta_{\mathrm{UD}}$ (mas) & $f_{\mathrm{UD}}(\%)$ & $f_{\text {free }}(\%)$ \\
\hline \multirow[t]{7}{*}{ PHOENIX } & 2016 & 1.53 & $4.4 \pm 0.2$ & 84.9 & $6.4 \pm 0.2$ & 12.3 & 2.8 \\
\hline & & 1.58 & $4.4 \pm 0.2$ & 86.6 & $6.3 \pm 0.2$ & 10.8 & 2.6 \\
\hline & & 1.63 & $4.4 \pm 0.2$ & 88.3 & $6.1 \pm 0.2$ & 8.4 & 3.3 \\
\hline & & 1.68 & $4.5 \pm 0.2$ & 94.2 & $12.3 \pm 0.2$ & 5.4 & 0.4 \\
\hline & & 1.72 & $4.4 \pm 0.2$ & 89.5 & $8.2 \pm 0.2$ & 9.8 & 0.7 \\
\hline & & 1.77 & $4.5 \pm 0.2$ & 88.7 & $8.3 \pm 0.2$ & 10.6 & 0.7 \\
\hline & & Average & $4.4 \pm 0.2$ & $88.7 \pm 3.2$ & $7.9 \pm 2.3$ & $9.5 \pm 2.4$ & $1.0 \pm 1.2$ \\
\hline \multirow[t]{7}{*}{ PHOENIX } & 2019 & 1.53 & $4.5 \pm 0.2$ & 93.0 & $11.1 \pm 0.2$ & 7.2 & 0.0 \\
\hline & & 1.58 & $4.6 \pm 0.2$ & 94.2 & $11.7 \pm 0.2$ & 6.0 & 0.0 \\
\hline & & 1.63 & $4.5 \pm 0.2$ & 91.2 & $6.7 \pm 0.2$ & 6.5 & 2.2 \\
\hline & & 1.68 & $4.5 \pm 0.2$ & 90.5 & $6.3 \pm 0.2$ & 7.5 & 2.0 \\
\hline & & 1.72 & $4.4 \pm 0.2$ & 82.7 & $5.7 \pm 0.2$ & 16.0 & 1.3 \\
\hline & & 1.77 & $4.4 \pm 0.2$ & 80.8 & $6.2 \pm 0.2$ & 17.4 & 1.8 \\
\hline & & Average & $4.5 \pm 0.2$ & $88.8 \pm 5.7$ & $8.0 \pm 2.7$ & $10.1 \pm 0.5$ & $1.1 \pm 1.0$ \\
\hline \multirow[t]{7}{*}{ 3D RHD 065} & 2016 & 1.53 & $4.1 \pm 0.2$ & 79.3 & $6.0 \pm 0.2$ & 17.9 & 2.8 \\
\hline & & 1.58 & $4.1 \pm 0.2$ & 82.2 & $6.1 \pm 0.2$ & 15.3 & 2.5 \\
\hline & & 1.63 & $4.2 \pm 0.2$ & 84.2 & $6.1 \pm 0.2$ & 12.8 & 3.0 \\
\hline & & 1.68 & $4.2 \pm 0.2$ & 88.2 & $7.2 \pm 0.2$ & 10.1 & 1.7 \\
\hline & & 1.72 & $4.2 \pm 0.2$ & 87.8 & $7.9 \pm 0.2$ & 11.5 & 0.7 \\
\hline & & 1.77 & $4.2 \pm 0.2$ & 86.7 & $7.9 \pm 0.2$ & 12.5 & 0.8 \\
\hline & & Average & $4.2 \pm 0.2$ & $84.7 \pm 3.5$ & $6.9 \pm 0.9$ & $13.4 \pm 2.8$ & $1.9 \pm 1.0$ \\
\hline \multirow[t]{7}{*}{ 3D RHD 067} & 2019 & 1.53 & $4.2 \pm 0.2$ & 86.3 & $5.9 \pm 0.2$ & 10.8 & 2.9 \\
\hline & & 1.58 & $4.3 \pm 0.2$ & 88.7 & $6.0 \pm 0.2$ & 8.9 & 2.4 \\
\hline & & 1.63 & $4.3 \pm 0.2$ & 89.7 & $6.7 \pm 0.2$ & 8.7 & 1.6 \\
\hline & & 1.68 & $4.3 \pm 0.2$ & 88.8 & $6.3 \pm 0.2$ & 9.3 & 1.9 \\
\hline & & 1.72 & $4.2 \pm 0.2$ & 76.4 & $5.5 \pm 0.2$ & 22.3 & 1.3 \\
\hline & & 1.77 & $4.2 \pm 0.2$ & 77.4 & $6.0 \pm 0.2$ & 21.1 & 1.5 \\
\hline & & Average & $4.2 \pm 0.2$ & $84.5 \pm 6.0$ & $6.1 \pm 0.4$ & $13.5 \pm 6.4$ & $1.9 \pm 0.6$ \\
\hline
\end{tabular}

Notes. $\Theta_{1}$ represents the Rosseland angular diameter in the case of the PHOENIX model, and the layer where $\mathrm{r} / \mathrm{R}_{\text {star }}=1$ in the 3D RHD model. $\Theta_{\mathrm{UD}}$ is the angular diameter of the uniform disk describing the MOLsphere. Finally, $f_{1}, f_{\mathrm{UD}}$, and $f_{\text {free }}$ describe the relative flux of the PHOENIX/3D RHD, MOLsphere, and free zero visibility scale components.

PROMETEOII/2014/057. We thank Karl-Heinz Hofmann for his help using the IRBis image reconstruction package.

\section{References}

Airapetian, V., Carpenter, K. G., \& Ofman, L. 2010, ApJ 723, 1210

Arroyo-Torres, B., Martí-Vidal, I., Marcaide, J. M., et al. 2014, A\&A. 566, A88

Arroyo-Torres, B., Wittkowski, M., Chiavassa, A., et al. 2015, A\&A 575, A50

Arroyo-Torres, B., Wittkowski, M., Marcaide, J. M., \& Hauschildt, P. H. 2013, A\&A 554, A76

Aurière, M., Donati, J. F., Konstantinova-Antova, R., et al. 2010, A\&A 516, L2

Baron, F., Monnier, J. D., \& Kloppenborg, B. 2010, in Society of Photo-Optical Instrumentation Engineers (SPIE) Conference Series, Vol. 7734, Optical and Infrared Interferometry II, 77342I

Chiavassa, A., Freytag, B., Masseron, T., \& Plez, B. 2011, A\&A |535, A22

Chiavassa, A., Haubois, X., Young, J. S., et al. 2010, A\&A 515, A12

Chiavassa, A., Plez, B., Josselin, E., \& Freytag, B. 2009, A\&A 506, 1351

Cranmer, S. R. \& Saar, S. H. 2011, ApJ 741, 54

De Beck, E., Decin, L., de Koter, A., et al. 2010, A\&A. 523, A18

Dessart, L., Hillier, D. J., Waldman, R., \& Livne, E. 2013, MNRAS. 433, 1745

Freytag, B. 2003, in Society of Photo-Optical Instrumentation Engineers (SPIE) Conference Series, Vol. 4838, Proc. SPIE, ed. W. A. Traub, 348-357

Freytag, B., Steffen, M., Ludwig, H. G., et al. 2012, Journal of Computational Physics. 231, 919

Groh, J. H., Meynet, G., Ekström, S., \& Georgy, C. 2014, A\&A 564, A30

Groh, J. H., Meynet, G., Georgy, C., \& Ekström, S. 2013, A\&A 558, A131

Hauschildt, P. H. \& Baron, E. 1999, Journal of Computational and Applied Mathematics, 109,41

Heger, A., Fryer, C. L., Woosley, S. E., Langer, N., \& Hartmann, D. H. 2003, ApJ 591, 288

Hofmann, K. H., Weigelt, G., \& Schertl, D. 2014, A\&A. 565, A48
Josselin, E. \& Plez, B. 2007, A\&A. 469, 671

Kravchenko, K., Chiavassa, A., Van Eck, S., et al. 2019, A\&A. 632, A28

Kravchenko, K., Van Eck, S., Chiavassa, A., et al. 2018, A\&A 610, A29

Lafrasse, S., Mella, G., Bonneau, D., et al. 2010, VizieR Online Data Catalog, II $/ 300$

Le Bouquin, J. B., Berger, J. P., Lazareff, B., et al. 2011, A\&A. 535, A67

Marco, A. \& Negueruela, I. 2013, A\&A. 552, A92

Meynet, G., Chomienne, V., Ekström, S., et al. 2015, A\&A 575, A60

Monnier, J. D. 2003, Reports on Progress in Physics 66, 789

Montargès, M., Kervella, P., Perrin, G., et al. 2016, A\&A. 588, A130

Montargès, M., Norris, R., Chiavassa, A., et al. 2018, A\&A. 614, A12

Paladini, C., Baron, F., Jorissen, A., et al. 2018, Nature 553, 310

Ragland, S., Le Coroller, H., Pluzhnik, E., et al. 2008, ApJ 679, 746

Rau, G., Ohnaka, K., Wittkowski, M., Airapetian, V., \& Carpenter, K. G. 2019, ApJ 882, 37

Smith, N. 2014, ARA\&A. 52, 487

Thirumalai, A. \& Heyl, J. S. 2012, MNRAS. 422, 1272

Tremblay, P. E., Ludwig, H. G., Freytag, B., Steffen, M., \& Caffau, E. 2013, A\&A. 557, A7

Tsuji, T. 2000, ApJ 540, L99

Vlemmings, W. H. T., Khouri, T., De Beck, E., et al. 2018, A\&A. 613, L4

Walmswell, J. J. \& Eldridge, J. J. 2012, MNRAS 419, 2054

Wang, Z., Bovik, A. C., Sheikh, H. R., Simoncelli, E. P., et al. 2004, IEEE transactions on image processing, 13, 13

Wittkowski, M., Abellán, F. J., Arroyo-Torres, B., et al. 2017a, A\&A 606, L1

Wittkowski, M., Arroyo-Torres, B., Marcaide, J. M., et al. 2017b, A\&A 597, A9 Wittkowski, M., Hauschildt, P. H., Arroyo-Torres, B., \& Marcaide, J. M. 2012, A\&A. 540, L12

Wittkowski, M., Hofmann, K. H., Höfner, S., et al. 2017c, A\&A 601, A3

Woillez, J., Abad, J. A., Abuter, R., et al. 2019, A\&A 629, A41

Yasuda, Y., Suzuki, T. K., \& Kozasa, T. 2019, ApJ 879, 77

Yoon, S.-C. \& Cantiello, M. 2010, ApJ 717, L62 


\section{Appendix A: Comparison of the pre-NAOMI 2016 and post-NAOMI 2019 PIONIER data}

We obtained pre-NAOMI PIONIER data in 2016 and post-NAOMI PIONIER data in 2019 during NAOMI Science Verification. The two data sets are very similar in terms of $u v$ coverage, time span, and atmospheric conditions. The total number of $u v$ points is 156 for both data sets, and their distribution is very similar (see Fig. 11. The 2016 data span 81 days, while those of 2019 span 70 days. The atmospheric conditions were similar (see Table 1) with an average seeing of 0.56 "and average coherence time of $4.3 \mathrm{msec}$ in 2016, and of $0.64^{\prime \prime}$ and $5.7 \mathrm{msec}$ in 2019. Both data sets used the same interferometric calibrator and the same observational and data reduction strategies.

The average error of the squared visibility amplitudes $\sigma\left(V^{2}\right) / V^{2}$ is $10.2 \%$ in 2016 and $5.6 \%$ in 2019 . The average error of the closure phases is $1.8^{\circ}$ in 2016 and $0.9^{\circ}$ in 2019 . The scatter of the visibility points, in particular at short baselines is much reduced in the 2019 data set compared to the 2016 data set (cf. Fig. 2). As otherwise the two data sets are very comparable, we attribute this improvement in precision and accuracy of the visibility data to the addition of the adaptive optics system NAOMI. Consequently, our average estimated pixel errors of the reconstructed images, based on different tests as outlined in detail in Appendix B, has improved from $17 \%$ to $14 \%$ for 2016 and 2019 , respectively.

\section{Appendix B: Estimation of image errors}

In order to ensure the validity of the substructures found in Fig. 3, we probed different systematic errors due to three effects:

i) Errors inside the SQUEEZE reconstruction package (see details in Appendix B.1);

ii) Errors due to the reconstruction package used (see details in Appendix B.2);

iii) Errors due to effects of our limited $u v$ coverage (see details in Appendix B.3.

\section{Appendix B.1: Errors within SQUEEZE}

To obtain the image that is shown in Fig. 3, we computed 50 different SQUEEZE images and averaged them. In a similar fashion to that used by Paladini et al. (2018), Fig. B.1] shows the images one standard deviation above (and below) the average image for 2016 and 2019 data. The persistence of the same features in these error images indicates that the substructures do not originate from the averaging procedure we followed.
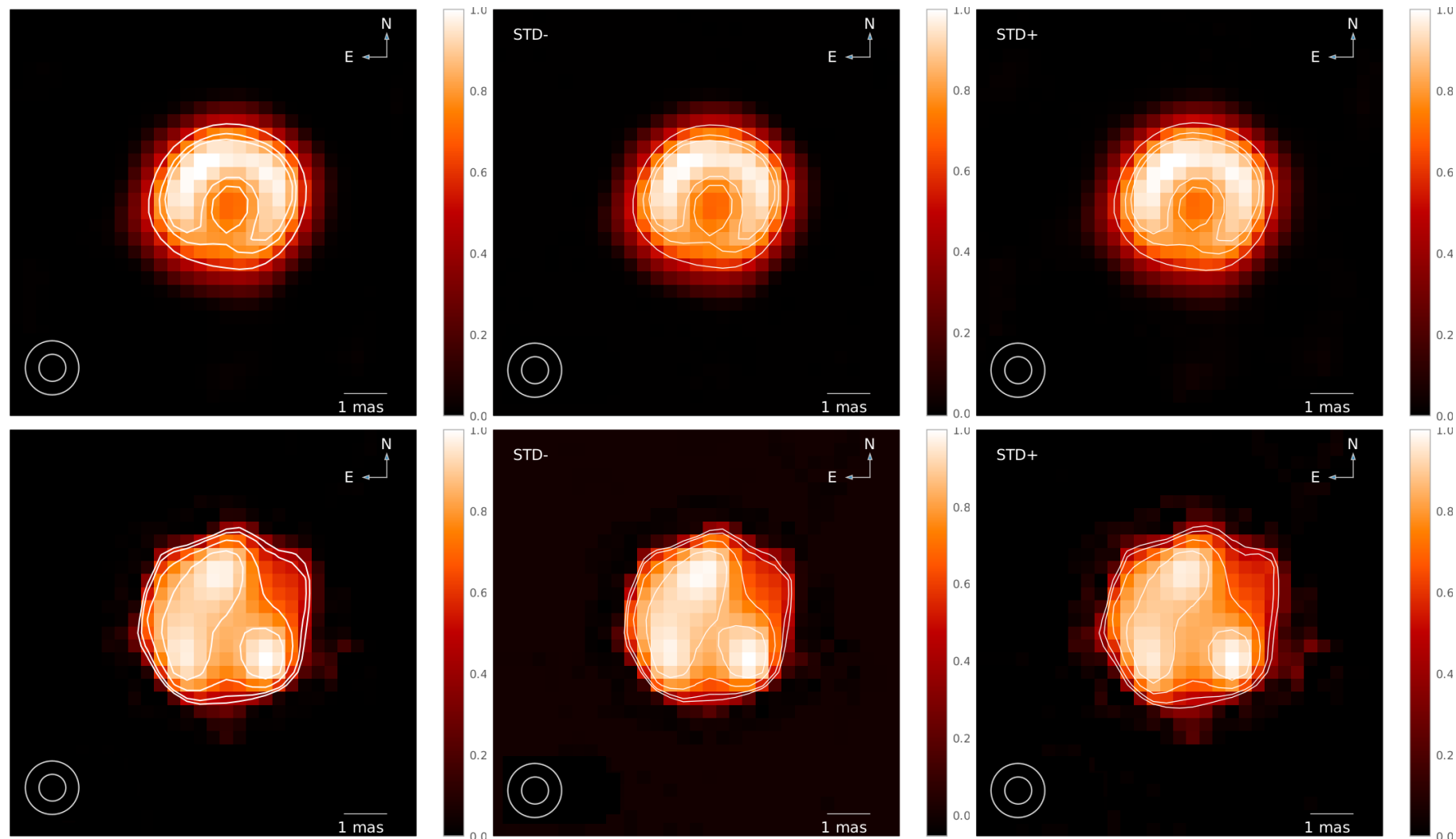

Fig. B.1. Squeeze average images (left column), images one standard deviation below the average (middle column), and images one standard deviation above the average (right column). The top row represents 2016 data (contours at 55\%, 77\%, and $85 \%$ of peak intensity), while the bottom row represents the 2019 data (contours at $40 \%, 50 \%, 70 \%$, and $87 \%$ of peak intensity). 


\section{Appendix B.2: IRBis reconstruction and comparison with SQUEEZE}

Following a similar method to that used for the carbon AGB star R Scl (Wittkowski et al.|2017c), we used the IRBis reconstruction package as follows:

i) We selected as start images the best-fit model from the PHOENIX + UD model discussed in Sect. 3

ii) We used a flat prior and the six available regularization functions of IRBis. For each regularization we tested reconstructions with decreasing values of the hyperparameter $\mu$ and increasing radii of the object mask.

iii) We chose as the final image that with the best quality derived from the $\chi^{2}$ values and residual ratio values of the visibility and closure phases $\left(q_{\text {rec }}\right.$ value). This image is based on regularization function 4 (edge preservation) in the 2016 and 2019 data.

Images obtained with regularization functions 1 (compactness), 3 (smoothness), 5 (smoothness), and 6 (quadratic Tikhonov), resulted in very similar images of similar quality parameters. Function 2 (maximum entropy) resulted in poorer reconstructions. The final images can be found in Fig. B.2.

For the same epoch, IRBis and SQUEEZE result in very similar images. Figure B.3 shows the difference between the two images (SQUEEZE - IRBis) evaluated pixel by pixel in terms of the flux of the SQUEEZE image. The results indicate that the same structures are present in the SQUEEZE and the IRBis reconstruction images, although some pixels differ in intensity value by up to $24 \%$. We applied a cut-off radius of 0.75 stellar radii (as stated in the main text) in order to avoid larger, non-physical errors near the limb of the star.

When comparing two astronomical images of the same object that only vary in the reconstruction method employed, the image differences need to originate in these methods. This scenario is equivalent to that which the SSIM was constructed for: a reference image (e.g., SQUEEZE reconstruction) and a distorted image with respect to its reference (e.g., IRBis reconstruction). The value obtained for these two reconstructed images is SSIM $=0.99$ in the 2016 and the 2019 data.

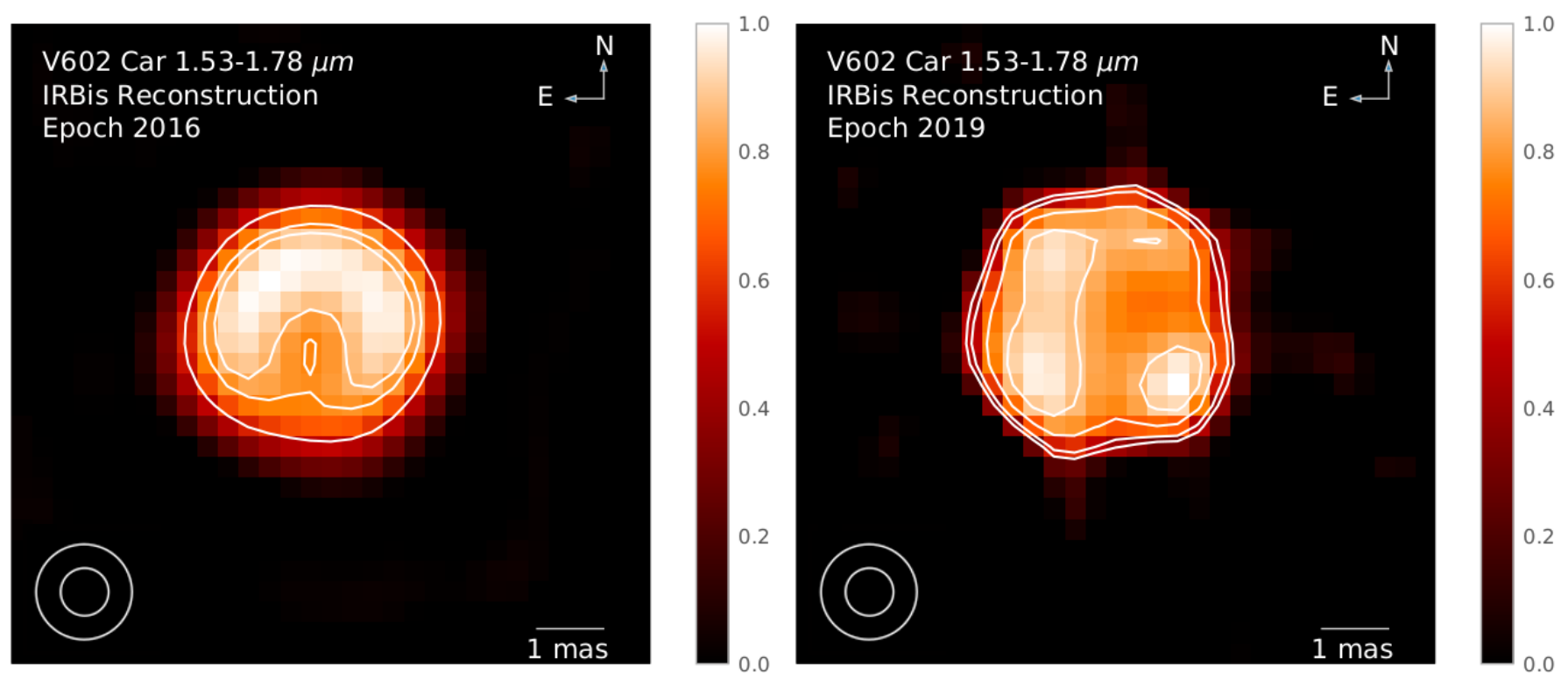

Fig. B.2. Same as Fig. 3 but for the IRBis reconstruction package. Contours are drawn at levels 55\%, 77\%, and $85 \%$ of the peak intensity in 2016 and at $40 \%, 50 \%, 70 \%$, and $87 \%$ of peak intensity in 2019 .

\section{Appendix B.3: Errors due to limited uv coverage}

A limited $u v$ coverage might produce artificial effects that could be concealed in the final image. In order to test for these effects, we simulated visibilities from the 3D RHD model snapshots using OITOOLS at the same $u v$ points of our observations. We added a typical amount of noise to the image and then reconstructed it using SQUEEZE in the usual way. Since we are interested in the validity of the surface features, no MOLsphere was added. We verified that an addition of a uniform MOLsphere, as modeled in Table 2, does not change the result.

Our results (see Fig. B.4) show that the reconstructions of the simulated visibilities (middle column of Fig. B.4) result in a very similar image to the original (left column of Fig. B.4), with an SSIM $=0.90$ in 2016 and SSIM $=0.89$ in 2019 . We computed these difference images with a convolved beam of $0.3,0.6$, and 1.2 mas and found that the resolution that best kept all the information about the substructures while reducing the errors is 0.6 mas, which is shown in Fig. B.4 Based on this analysis, we can estimate the super-resolution that we achieve with our $u v$ coverage and our noise to 0.6 mas, compared to the nominal resolution $\lambda /\left(2 B_{\max }\right)$ of 1.2 mas.

From this discussion, we conclude that the substructures found in Fig. 3 are probably not caused or altered by the limited $u v$ coverage of our observations. 

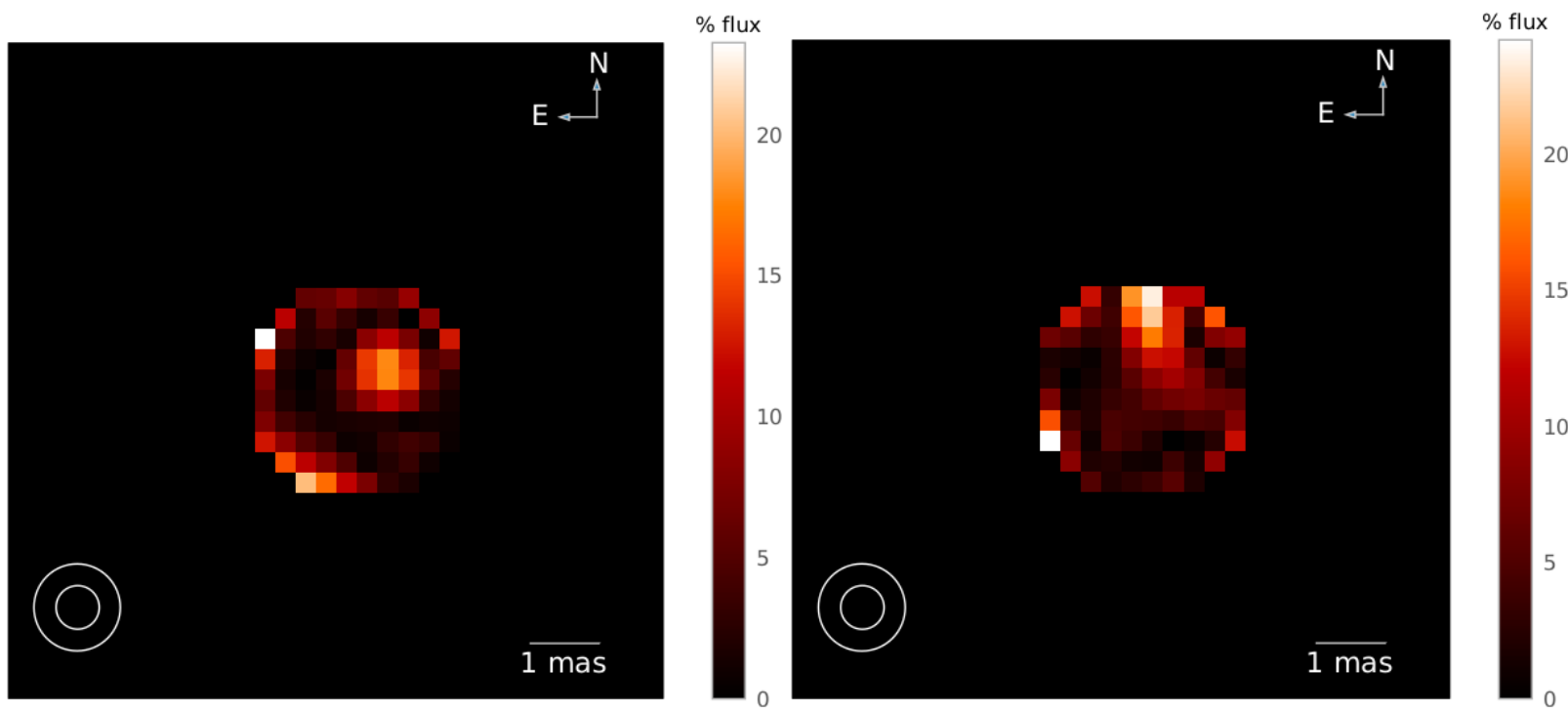

Fig. B.3. Image resulting from the pixel-by-pixel difference between SQUEEZE and IRBis reconstructions shown in Fig. 3 and Fig. B.2 (Left): 2016 epoch. (Right): 2019 epoch. The scale represents the percentage of flux of the original SQUEEZE image. The same cut-off radius is applied as presented in the main text: 0.75 stellar radii.
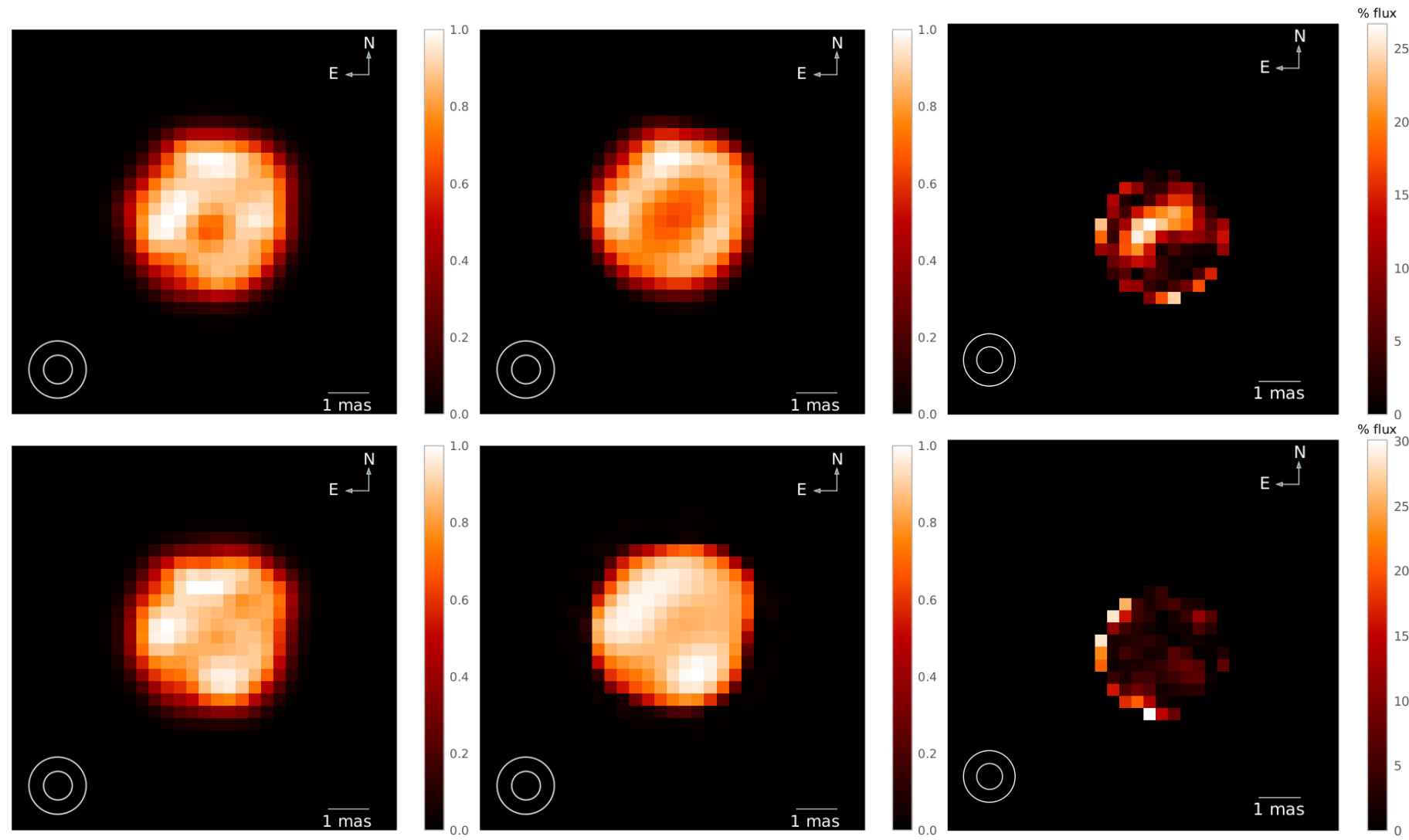

Fig. B.4. Original snapshot image convolved to 0.6 mas resolution (left column), SQUEEZE reconstructed image from the synthetic visibilities of the left image (middle column; not further convolved beyond the pixel scale of 0.3 mas/pixel), and pixel-by-pixel difference image between left column and middle column images in terms of the original flux (right column). Top panel for snapshot 65 and bottom panel for snapshot 67. No rotation of the images was applied here. The same cut-off radius is applied as presented in the main text: 0.75 stellar radii. 


\section{Appendix C: Spectral channel images of V602 Car}

\section{Appendix D: Mathematical definition of the SSIM}

When calculated on various image windows $(x$ and $y)$ of the same size $(\mathrm{N} \times \mathrm{N})$, the SSIM index is computed:

$\operatorname{SSIM}(x, y)=\frac{\left(2 \mu_{x} \mu_{y}+c_{1}\right)\left(2 \sigma_{x y}+c_{2}\right)}{\left(\mu_{x}^{2}+\mu_{y}^{2}+c_{1}\right)\left(\sigma_{x}^{2}+\sigma_{y}^{2}+c_{2}\right)}$,

where

$\mu_{x}$ is the average value of $\mathrm{x}$;

$\mu_{y}$ is the average value of $\mathrm{y}$;

$\sigma_{x}^{2}$ is the variance of $\mathrm{x}$;

$\sigma_{y}^{2}$ is the variance of $\mathrm{y}$;

$\sigma_{x y}$ is the covariance of $\mathrm{x}$ and $\mathrm{y}$;

$c_{1}=\left(k_{1} L\right)^{2}, c_{2}=\left(k_{2} L\right)^{2}$ are two variables to stabilize the division with weak denominator;

$L$ represents the dynamic range of the pixel values and is determined by the number of levels of luminance per pixel.

By default, $k_{1}=0.01$ and $k_{2}=0.03$. 

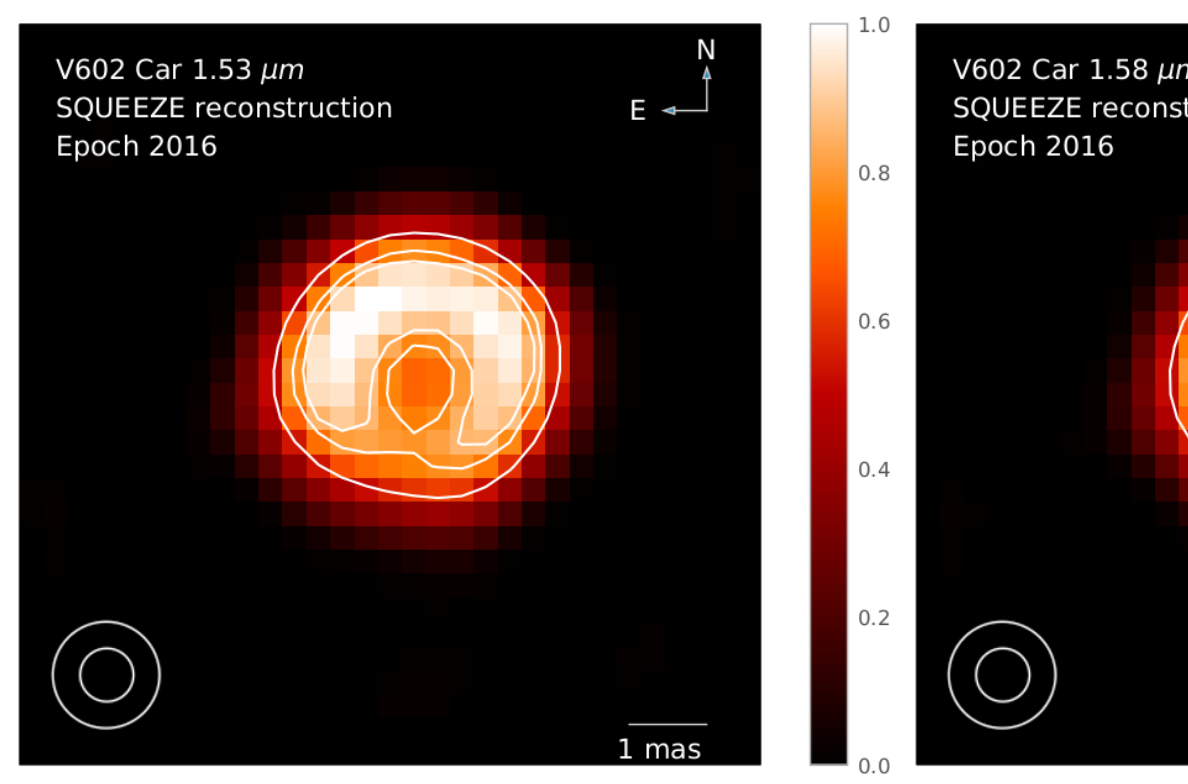

SQUEEZE reconstruction

Epoch 2016
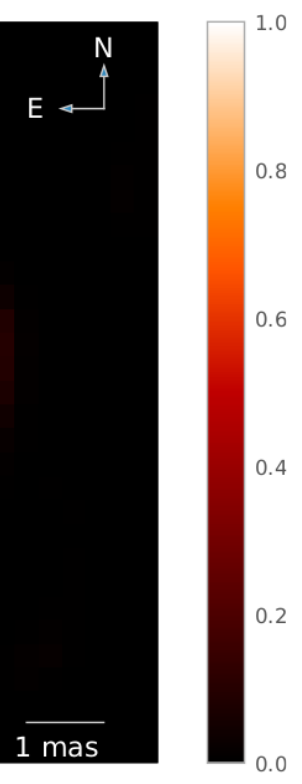

V602 Car $1.63 \mu \mathrm{m}$

SQUEEZE reconstruction

Epoch 2016
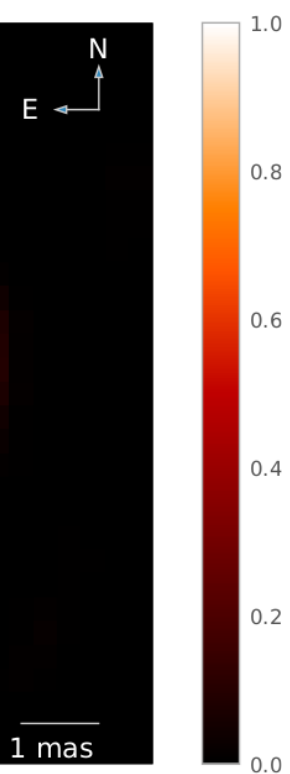

V602 Car $1.68 \mu \mathrm{m}$

SQUEEZE reconstruction

Epoch 2016

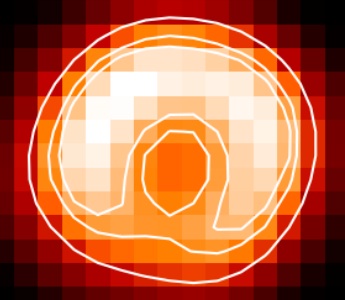

1 mas

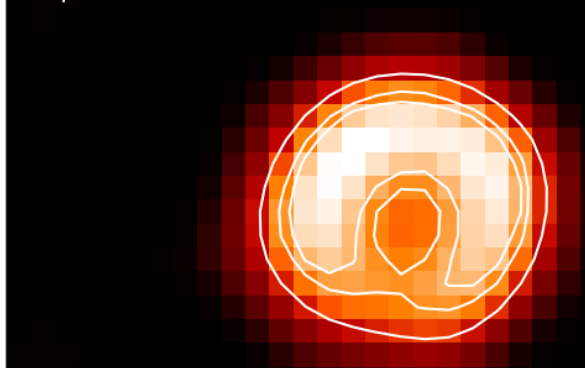

1 mas

V602 Car $1.72 \mu \mathrm{m}$

SQUEEZE reconstruction

Epoch 2016

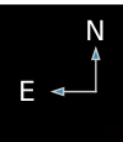

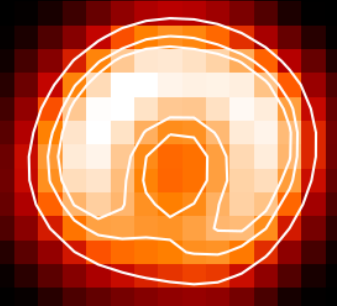

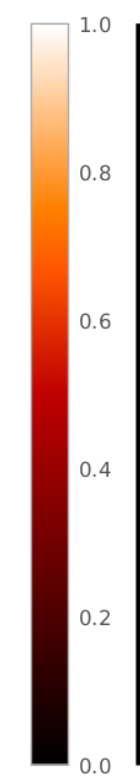

V602 Car $1.77 \mu \mathrm{m}$

SQUEEZE reconstruction

Epoch 2016
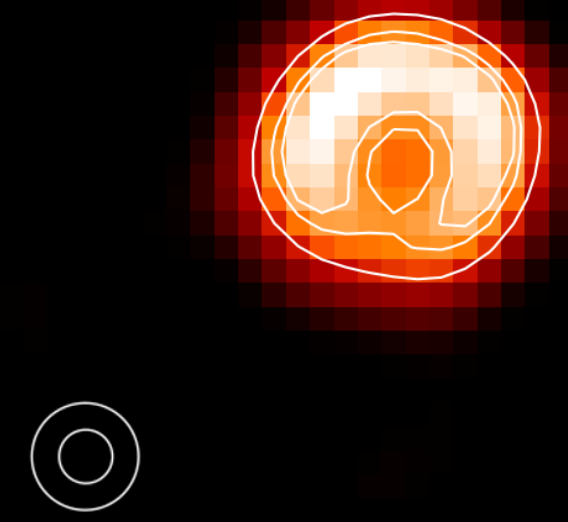

mas

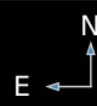

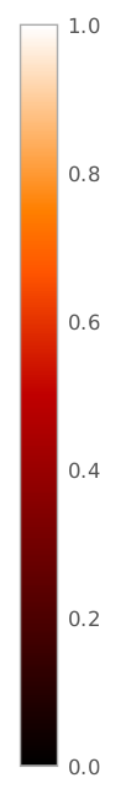

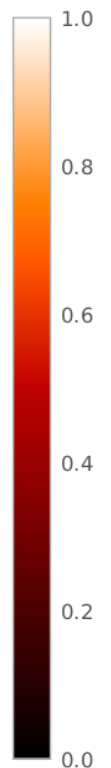

Fig. C.1. SQUEEZE reconstruction of 2016 V602 Car data at each spectral channel. Contours are drawn at levels 55\%, $77, \%$ and $85 \%$ of the peak intensity. 


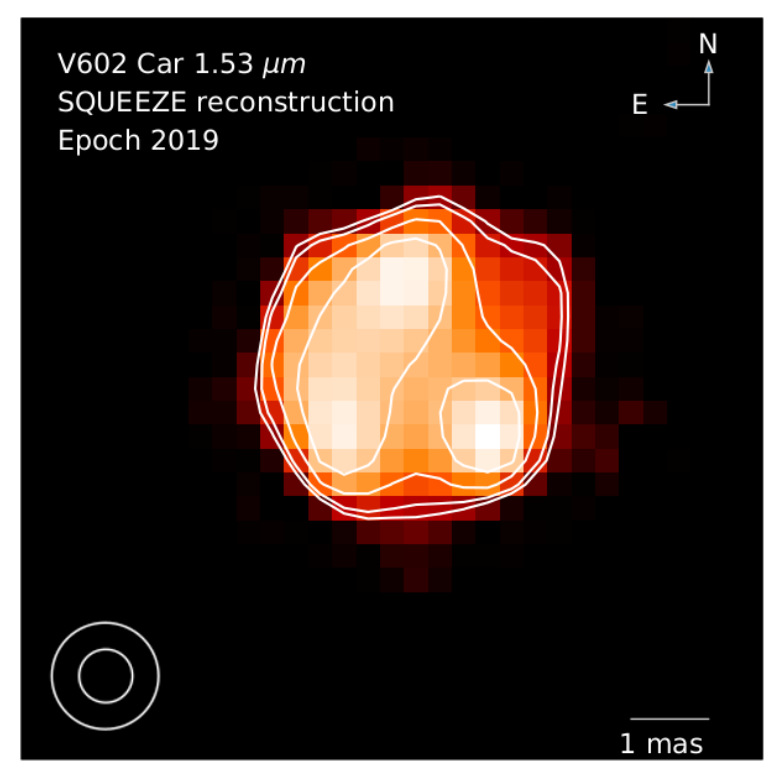

V602 Car $1.63 \mu \mathrm{m}$

SQUEEZE reconstruction

Epoch 2019
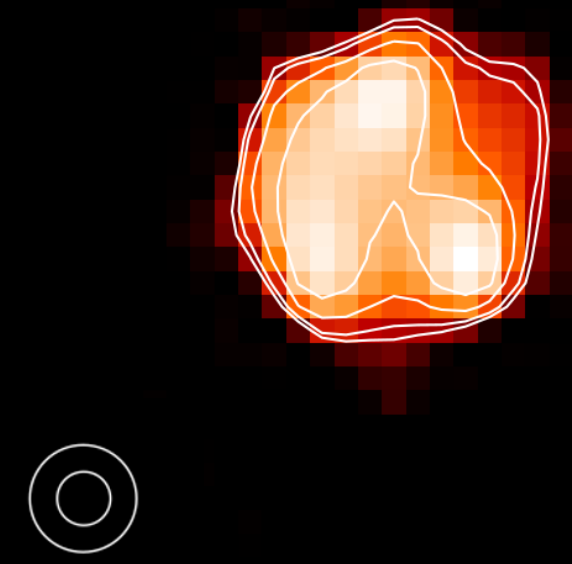

1 mas

\section{V602 Car $1.72 \mu \mathrm{m}$}

SQUEEZE reconstruction

Epoch 2019
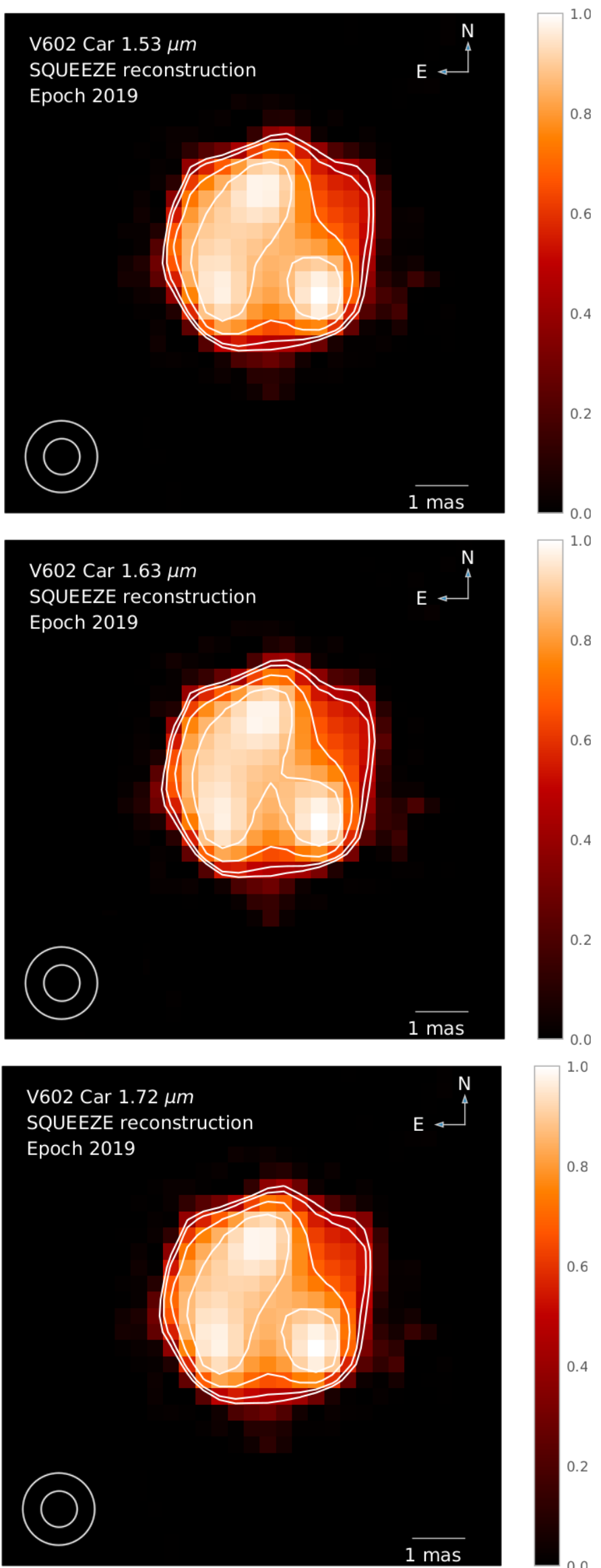

$E \longleftarrow$
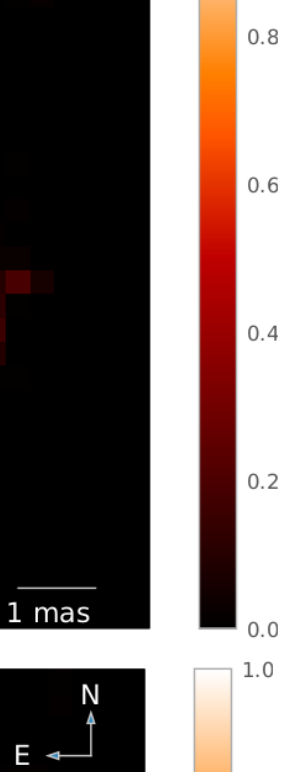

0.8

0.6

0.4

0.2

\section{V602 Car $1.58 \mu \mathrm{m}$}

SQUEEZE reconstruction

Epoch 2019
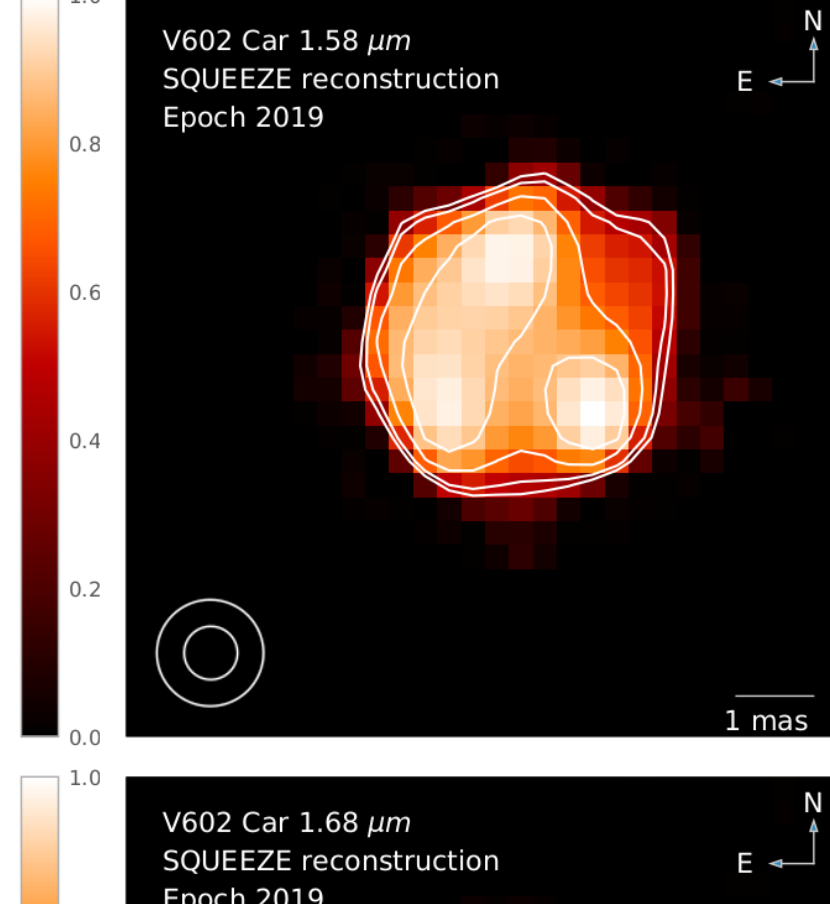

V602 Car $1.68 \mu \mathrm{m}$

SQUEEZE reconstruction

Epoch 2019

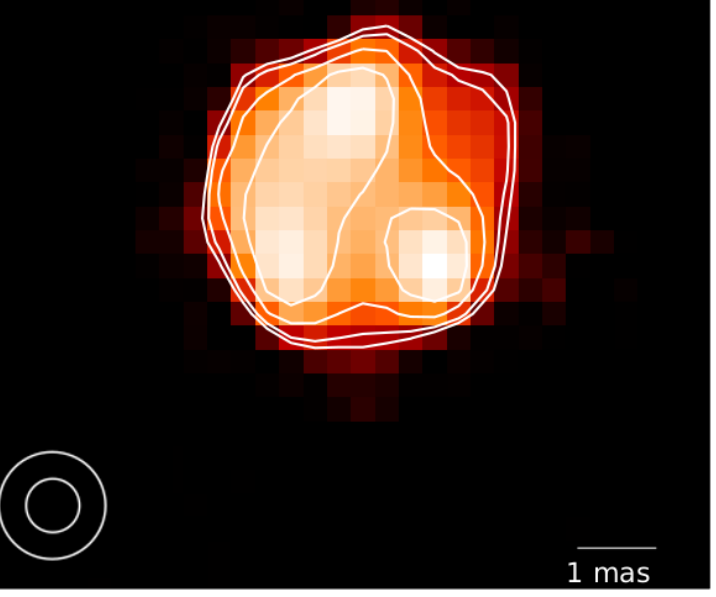

V602 Car $1.77 \mu \mathrm{m}$

SQUEEZE reconstruction

Epoch 2019

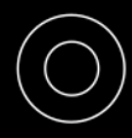

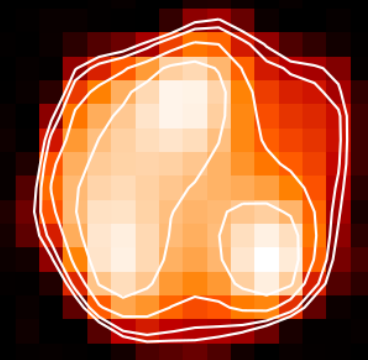

1 mas
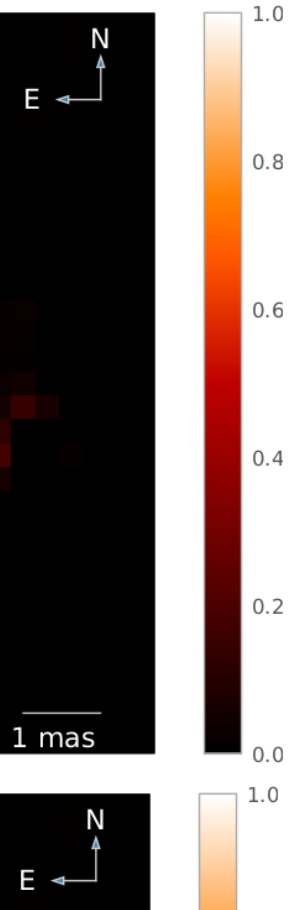

(1)

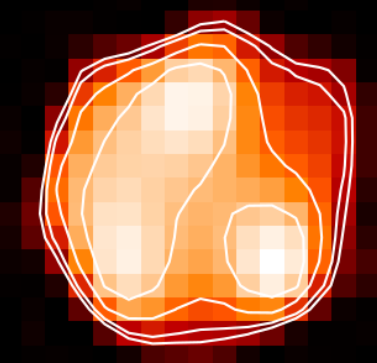

Fig. C.2. SQUEEZE reconstruction of 2019 V602 Car data at each spectral channel. Contours are drawn at levels $40 \%$, 50\%, 70\%, and $87 \%$ of the peak intensity. 